\title{
Generalized Blocks of Unipotent Characters in the Finite General Linear Group
}

\author{
Jean-Baptiste Gramain \\ École Polytechnique Fédérale de Lausanne \\ Lausanne, Switzerland \\ jean-baptiste.gramain@epfl.ch
}

July, 2007

\begin{abstract}
In a paper of 2003, B. Külshammer, J. B. Olsson and G. R. Robinson defined $\ell$-blocks for the symmetric groups, where $\ell>1$ is an arbitrary integer, and proved that they satisfy an analogue of the Nakayama Conjecture. Inspired by this work and the definitions of generalized blocks and sections given by the authors, we give in this paper a definition of $d$-sections in the finite general linear group, and construct $d$-blocks of unipotent characters, where $d \geq 1$ is an arbitrary integer. We prove that they satisfy one direction of an analogue of the Nakayama Conjecture, and, in some cases, prove the other direction. We also prove that they satisfy an analogue of Brauer's Second Main Theorem.
\end{abstract}

\section{Introduction, generalized blocks}

Throughout this paper, we let $n$ be a positive integer and $q$ be a power of a prime $p$. We let $V$ be an $n$-dimensional vector space over a finite field $\mathbf{F}_{q}$ with $q$ elements. We will work in the finite general linear group $G=G L(V)$. It will sometimes be convenient to choose a basis for $V$, and then identify $G$ with the group $G L(n, q)$ of invertible $n$ by $n$ matrices with entries in $\mathbf{F}_{q}$. The irreducible complex characters of $G$ have been described by J. A. Green in [6], using deep combinatorial arguments. Then, using in particular the Deligne-Lusztig theory, P. Fong and B. Srinivasan have classified the blocks of $G$ (cf [5]). The unipotent characters of $G$ are parametrized by the partitions of $n$. It turns out that, if $r$ is a prime not dividing $q$, then two unipotent characters belong to the same $r$-block of $G$ if and only if the partitions labeling them have the same $e$-core, where $e$ is the multiplicative order of $q$ modulo $r$. This result is shown using analogues of the Murnaghan-Nakayama rule for irreducible characters of $G$. Our aim is to use these analogues to obtain properties of some generalized blocks we define in $G$, and which don't depend on any prime. We construct unipotent blocks for $G$ which satisfy one direction of an analogue of the Nakayama Conjecture. In some cases, we also prove that they satisfy both directions.

The concept of generalized blocks was introduced by B. Külshammer, J. B. Olsson and G. R. Robinson ( $\mathrm{cf}[\underline{8})$. Take any finite group $H$, and take a union $\mathcal{C}$ 
of conjugacy classes of $H$ containing the identity. We can consider the restriction to $\mathcal{C}$ of the ordinary scalar product on characters of $H$. We denote by $\operatorname{Irr}(H)$ the set of complex irreducible characters of $H$. For $\chi, \psi \in \operatorname{Irr}(H)$, we let

$$
<\chi, \psi>_{\mathcal{C}}:=\frac{1}{|H|} \sum_{h \in \mathcal{C}} \chi(h) \psi\left(h^{-1}\right) .
$$

Then $\chi$ and $\psi$ are said to be directly $\mathcal{C}$-linked if $\left\langle\chi, \psi>_{\mathcal{C}} \neq 0\right.$, and orthogonal across $\mathcal{C}$ otherwise. Then direct $\mathcal{C}$-linking is a reflexive (since $1 \in \mathcal{C}$ ) and symmetric binary relation on $\operatorname{Irr}(H)$. Extending it by transitivity, we obtain an equivalence relation (called $\mathcal{C}$-linking) on $\operatorname{Irr}(H)$ whose equivalence classes are called the $\mathcal{C}$-blocks. Note that, since they are orthogonal on the whole of $H$, two distinct irreducible characters are directly linked across $\mathcal{C}$ if and only if they are directly linked across $H \backslash \mathcal{C}$. Note also that, if we take $\mathcal{C}$ to be the set of $r$-regular elements of $H$, for some prime $r$, then the $\mathcal{C}$-blocks are just the $r$-blocks (cf e.g. 10]).

In 8 , the authors have defined $\ell$-blocks for the symmetric groups, where $\ell \geq 2$ is any integer. To obtain this, they take $\mathcal{C}$ to be the set of $\ell$-regular elements, i.e. none of whose cycle has length divisible by $\ell$ (in particular, if $\ell$ is a prime $r$, then the $\ell$-blocks are just the $r$-blocks). The $\ell$-blocks thus obtained satisfy an analogue of the Nakayama Conjecture: two irreducible characters $\varphi_{\lambda}$ and $\varphi_{\mu}$ of the symmetric group $S_{n}$ (where $\lambda$ and $\mu$ are partitions of $n$ ) belong to the same $\ell$-block if and only if $\lambda$ and $\mu$ have the same $\ell$-core.

Following this work, A. Maróti studied generalized blocks in the alternating groups, and proved that, if $\ell$ is 2 or any odd integer greater than 1 , then the $\ell$-blocks of the alternating groups also satisfy an analogue of the Nakayama Conjecture (cf [9]).

In the case of symmetric groups, the argument for one of the directions goes as follows. Any element of a symmetric group can be written uniquely as a disjoint product of an $\ell$-regular element and an $\ell$-element (i.e. all of whose cycles have length divisible by $\ell$ ). Two elements are said to belong to the same $\ell$-section if their $\ell$-parts are conjugate. The $\ell$-section of the identity is thus the set of $\ell$-regular elements. The $\ell$-sections have properties very similar to those of ordinary prime sections. Even though we don't need sections in order to construct generalized blocks, they are important when one wants to discuss properties of blocks such as analogues of Brauer's Second Main Theorem.

If $x$ is an $h$-cycle of the symmetric group $S_{n}$ and $y \in S_{n}$ fixes the points moved by $x$ (and can thus be seen as an element of $S_{n-h}$ ), then the MurnaghanNakayama Rule allows us the write the value of a character $\varphi_{\lambda}$ of $S_{n}$ at $x y$ as a linear combination of the $\varphi_{\mu}(y)$ 's, where $\mu$ runs through the set of partitions of $n-h$ which can be obtained by removing an $h$-hook from $\lambda$.

Repeated use of this rule allows us to remove all of the $\ell$-part of an element by removing a sequence of $\ell$-hooks from the partition labeling the character we consider. An induction argument then proves that, if two characters of $S_{n}$ are linked across any $\ell$-section, then the partitions labeling them must have the same $\ell$-core.

Now, in $G=G L(V)$, we don't have such a nice cycle structure anymore. However, we can use the rational canonical form, which is based on the elementary divisors of a given element $g$. These are polynomials over $\mathbf{F}_{q}$, and 
they correspond to the cyclic subspaces of $V$ under the action of $g$. Then, very much like in the symmetric group, the Murnaghan-Nakayama Rule for unipotent characters allows us to relate the "removal" of a cyclic subspace associated to a polynomial of degree $d$ to the removal of $d$-hooks from the partition labeling the unipotent character we consider.

The definitions of $d$-element and $d$-regular element we give are such that we can apply the Murnaghan-Nakayama Rule and show by an induction argument that, if two unipotent characters are linked across any $d$-section, then the partitions labeling them have the same $d$-core.

In section 2, we present the conjugacy classes of $G L(V)$ and the primary decomposition, which is the basis for the definitions we introduce. In section 3 , we define the $d$-sections we work on, and the generalized blocks they define. Section 4 is devoted to the analogue of the Nakayama Conjecture for unipotent $d$-blocks. We prove that one direction is true in general, and that the other is true for $d=1$ and, for any $d>0$, for blocks of weight at most $(d+1) / 2$. We conjecture that, in fact, these restrictions are not necessary. Finally, in section 5 , we discuss an analogue of Brauer's Second Main Theorem for unipotent $d$ blocks.

\section{Conjugacy classes, primary decomposition}

\subsection{Rational canonical form}

We first introduce the theory of elementary divisors and the rational canonical form in $G$, which gives a parametrization of the conjugacy classes. For the results we give in this section, we refer to Green [6.

For any non-negative integer $k$, we will write $\nu \vdash k$ to say that $\nu$ is a partition of $k$, and we will write $|\nu|=k$. We denote by $\mathcal{P}$ the set of all partitions. We write $\mathcal{F}=\left\{f_{i}, i \in \mathcal{I}\right\}$ the set of irreducible, monic polynomials distinct from $X$ and of degree at most $n$ in $\mathbf{F}_{q}[X]$ (so that $\mathcal{I}$ is finite). For $f \in \mathcal{F}$, we write $\delta(f)$ for the degree of $f$. Then the conjugacy classes of $G$ are parametrized by the set of partition-valued functions $\zeta: \mathcal{F} \longrightarrow \mathcal{P}$ such that $\sum_{f \in \mathcal{F}}|\zeta(f)| \delta(f)=n$. For such a $\zeta$, the corresponding conjugacy class of $G$ will be written $\left(f_{1}^{\nu_{1}}, \ldots, f_{r}^{\nu_{r}}\right)$, where $\left\{f_{1}, \ldots, f_{r}\right\}=\{f \in \mathcal{F},|\zeta(f)| \neq 0\}$, and, for $1 \leq i \leq r, \nu_{i}=\zeta\left(f_{i}\right)$. We will sometimes also use the notation $\left(f^{\zeta(f)}\right)_{f \in \mathcal{F}}$ (thus allowing $|\zeta(f)|$ to be 0 ).

Take a conjugacy class $c=\left(f_{1}^{\nu_{1}}, \ldots, f_{r}^{\nu_{r}}\right)$ of $G$, and $g \in c$. Then, the characteristic polynomial of $g$ over $\mathbf{F}_{q}$ is

$$
\operatorname{Char}(g)=\prod_{i=1}^{r} f_{i}^{\left|\nu_{i}\right|} .
$$

Writing, for each $i, \nu_{i}=\left(\lambda_{i, 1} \geq \lambda_{i, 2} \geq \ldots \geq \lambda_{i, s}\right)$ (where $s$ can be chosen to be big enough for all $f_{i}^{\prime} s$ appearing in $\operatorname{Char}(g)$ (and even for all $g \in G$ ), by taking for example $s=n$ ), the minimal polynomial of $g$ over $\mathbf{F}_{q}$ is

$$
\operatorname{Min}(g)=\prod_{i=1}^{r} f_{i}^{\lambda_{i, 1}} .
$$


For $k=1, \ldots, s$, the polynomial $E D_{k}(g)=\prod_{i=1}^{r} f_{i}^{\sum_{j=1}^{k} \lambda_{i, j}}$ is the $k$-th elementary divisor of $g$ over $\mathbf{F}_{q}$.

For any irreducible monic polynomial $f$ over $\mathbf{F}_{q}$, we let $U(f)$ be the companion matrix of $f$, i.e. if $f(X)=X^{d}-a_{d-1} X^{d-1}-\cdots-a_{0}$, then

$$
U(f)=U_{1}(f)=\left(\begin{array}{ccccc}
0 & 1 & & & \\
\vdots & \ddots & \ddots & (0) & \\
\vdots & & \ddots & \ddots & \\
0 & \cdots & \cdots & 0 & 1 \\
a_{0} & a_{1} & \cdots & \cdots & a_{d-1}
\end{array}\right)
$$

For any positive integer $\lambda$, we write

$$
U_{\lambda}(f)=\left(\begin{array}{ccccc}
U(f) & I_{d} & & & \\
& U(f) & I_{d} & & \\
& & \ddots & \ddots & \\
& & & \ddots & I_{d} \\
& & & & U(f)
\end{array}\right),
$$

where $I_{d}$ is the $d$ by $d$ identity matrix, and there are $\lambda$ blocks $U(f)$ on the diagonal. Note that $U_{\lambda}(f)$ is equivalent over $\mathbf{F}_{q}$ to the companion matrix of $f^{\lambda}$. Finally, for $\nu=\left(\lambda_{1} \geq \ldots \geq \lambda_{s}\right)$ any partition (of an integer $k$ say), we write

$$
U_{\nu}(f)=\left(\begin{array}{ccc}
U_{\lambda_{1}}(f) & & \\
& \ddots & \\
& & U_{\lambda_{s}}(f)
\end{array}\right),
$$

where $U_{0}(f)$ has to be seen as the empty matrix. If $g \in c=\left(f_{1}^{\nu_{1}}, \ldots, f_{r}^{\nu_{r}}\right)$, then, in any matrix representation of $G, g$ is equivalent over $\mathbf{F}_{q}$ to

$$
U_{\left(f_{1}^{\nu_{1}}, \ldots, f_{r}^{\nu_{r}}\right)}=\left(\begin{array}{ccc}
U_{\nu_{1}}\left(f_{1}\right) & & \\
& \ddots & \\
& & U_{\nu_{r}}\left(f_{r}\right)
\end{array}\right) .
$$

This is the rational canonical form of $g$ over $\mathbf{F}_{q}$.

\subsection{The Jordan decomposition}

An element $g \in G$ is semisimple if and only if it is diagonalizable over an algebraic extension of $\mathbf{F}_{q}$ (i.e. some $\mathbf{F}_{q^{k}}, k \geq 1$ ), i.e. $\operatorname{Min}(g)$ splits over $\mathbf{F}_{q^{k}}$ and has only simple roots. Equivalently, $g \in G L(V)$ is semisimple if and only if there is a basis of $V$ consisting of eigenvectors of $g$. Let $c$ be the conjugacy class of $g$. If $c=\left(f_{1}^{\nu_{1}}, \ldots, f_{r}^{\nu_{r}}\right)$, then $g$ is semisimple if and only if, for all $1 \leq i \leq r$, we have $\nu_{i}=(1, \ldots, 1)$.

Choose some basis for $V$. We write $\Delta\left(\left(q_{i}\right)\right)$ for the diagonal matrix with diagonal blocks the $q_{i}$ 's. Suppose $g=\Delta\left(\left(U_{\nu_{i}}\left(f_{i}\right)\right)\right)$. Then $g=g_{S}+g_{N}=$ 
$\Delta\left(\left(U_{\tilde{\nu}_{i}}\left(f_{i}\right)\right)\right)+\Delta\left(\left(\tilde{I}_{\nu_{i}}\left(f_{i}\right)\right)\right)$, where $\tilde{\nu_{i}}=(1, \ldots, 1) \vdash\left|\nu_{i}\right|=k_{i}$ and

$$
\tilde{I}_{\nu_{i}}\left(f_{i}\right)=\left(\begin{array}{ccccc}
(0) & I_{d_{i}} & & & \\
& (0) & I_{d_{i}} & & \\
& & \ddots & \ddots & \\
& & & \ddots & I_{d_{i}} \\
& & & & (0)
\end{array}\right)
$$

with $\left|\nu_{i}\right|=k_{i}$ diagonal $d_{i}$ by $d_{i}$ blocks, where $f_{i}$ has degree $d_{i}$.

Thus $g_{S}=\Delta\left(\left(U_{k_{i}}\left(f_{i}\right)\right)\right)$ is the semisimple part of $g$, and the unipotent part of $g$ is $g_{U}=I_{n}+g_{S}^{-1} g_{N}$.

Now, if $\tilde{g}=h g h^{-1}$ for some $h \in G$, then $\tilde{g}=\left(h g_{S} h^{-1}\right)\left(h g_{U} h^{-1}\right)$, and we see that the semisimple and unipotent parts of $\tilde{g}$ are $h g_{S} h^{-1}$ and $h g_{U} h^{-1}$ respectively.

\subsection{Primary decomposition}

We first mention a fact about centralizers in $G L(V)$. Take $g \in G L(V)$. If, in some matrix representation corresponding to the decomposition $V=V_{1} \oplus V_{2}$, we have

$$
g=\left(\begin{array}{cc}
g_{1} & \\
& g_{2}
\end{array}\right)
$$

with, for $i \in\{1,2\}, g_{i} \in G_{i}=G L\left(V_{i}\right)$, and $\operatorname{gcd}\left(\operatorname{Min}\left(g_{1}\right), \operatorname{Min}\left(g_{2}\right)\right)=1$, then

$$
C_{G}(g)=\left\{\left(\begin{array}{ll}
a & \\
& b
\end{array}\right), a \in C_{G_{1}}\left(g_{1}\right), b \in C_{G_{2}}\left(g_{2}\right)\right\},
$$

and thus $C_{G}(g) \cong C_{G_{1}}\left(g_{1}\right) \times C_{G_{2}}\left(g_{2}\right)$ via $h \longmapsto\left(\left.h\right|_{V_{1}},\left.h\right|_{V_{2}}\right)$ (which is independant on the matrix representation).

We also give the following lemma

Lemma 2.1. Let $g \in G=G L(V)$. Suppose $V_{1}$ and $V_{2}$ are $g$-stable subspaces of $V$ such that $V=V_{1} \oplus V_{2}$. If $\left.g\right|_{V_{1}} \in\left(f^{\zeta_{1}(f)}\right)_{f \in \mathcal{F}}$ and $\left.g\right|_{V_{2}} \in\left(f^{\zeta_{2}(f)}\right)_{f \in \mathcal{F}}$ for some $\zeta_{1}, \zeta_{2}: \mathcal{F} \longrightarrow \mathcal{P}$, then $g \in\left(f^{\zeta_{1}(f) \cup \zeta_{2}(f)}\right)_{f \in \mathcal{F}}$, where, by $\cup$, we denote the concatenation of partitions (i.e., for partitions $\mu$ and $\nu$, the components of the partition $\mu \cup \nu$ are those of $\mu$ together with those of $\nu$. If $\mu \vdash k$ and $\nu \vdash \ell$, then $\mu \cup \nu \vdash k+\ell$.)

Proof. Taking any bases for $V_{1}$ and $V_{2}$ (which then add to give a basis of $V$ ), we can write

$$
g=\left(\begin{array}{ll}
g_{1} & \\
& g_{2}
\end{array}\right)
$$

(where $g_{1}$ (resp. $\left.g_{2}\right)$ is the matrix of $\left.g\right|_{V_{1}}$ (resp. $\left.\left.g\right|_{V_{2}}\right)$ ). The idea is that we can obtain the rational canonical form of $g$ by reducing to this form $g_{1}$ and $g_{2}$. There exist $h_{1} \in G L\left(V_{1}\right)$ and $h_{2} \in G L\left(V_{2}\right)$ such that

$$
g=\left(\begin{array}{ll}
h_{1} & \\
& h_{2}
\end{array}\right)^{-1}\left(\begin{array}{ll}
\left.U_{\left(f \zeta_{1}(f)\right.}\right)_{f \in \mathcal{F}} & \\
& \left.U_{\left(f \zeta_{2}(f)\right.}\right)_{f \in \mathcal{F}}
\end{array}\right)\left(\begin{array}{ll}
h_{1} & \\
& h_{2}
\end{array}\right) .
$$


Thus, for some permutation matrix $P$,

$$
g=\left(\begin{array}{ll}
h_{1} & \\
& h_{2}
\end{array}\right)^{-1} P^{-1} U_{\left(f \zeta_{1}(f) \cup \zeta_{2}(f)\right)_{f \in \mathcal{F}}} P\left(\begin{array}{ll}
h_{1} & \\
& h_{2}
\end{array}\right),
$$

so that $g \in\left(f^{\zeta_{1}(f) \cup \zeta_{2}(f)}\right)_{f \in \mathcal{F} \text {. }}$

We now turn to the primary decomposition of elements of $G$. Take $g \in G L(V)$ and suppose $g \in\left(f_{1}^{\nu_{1}}, \ldots, f_{r}^{\nu_{r}}\right)$. Then there exists a unique decomposition $V=V_{1} \oplus \cdots \oplus V_{r}$, where the $V_{i}$ 's are $g$-stable subspaces of $V$ and, for $1 \leq i \leq r,\left.g\right|_{V_{i}} \in\left(f_{i}^{\nu_{i}}\right) \subset G L\left(V_{i}\right)$. For each $1 \leq i \leq r, V_{i}$ is given by

$$
V_{i}=\left\{v \in V \mid f_{i}^{k}(g) v=0 \text { for some } k>0\right\} .
$$

We have $C_{G}(g) \cong C_{G L\left(V_{1}\right)}\left(\left.g\right|_{V_{1}}\right) \times \cdots \times C_{G L\left(V_{r}\right)}\left(\left.g\right|_{V_{r}}\right)$. Then there exists a unique writing $g=g_{1} \ldots g_{r}$, where, for each $1 \leq i \leq r, g_{i} \in G L(V), V_{i}$ is $g_{i}$-stable and $\left.g_{i}\right|_{V_{j}}=1$ for all $j \neq i$. Indeed, we must have $\left.g_{i}\right|_{V_{i}}=\left.g\right|_{V_{i}}$ for each $1 \leq i \leq r$, and $g_{i}$ is uniquely determined by this and its properties listed before. Furthermore, the $g_{i}$ 's are pairwise commuting elements. We say that $g_{1} \ldots g_{r}$ is the primary decomposition of $\mathrm{g}$. The $g_{i}$ 's are the primary components of $g$.

More generally, an element of $G$ is said to be primary if its characteristic polynomial is divisible by at most one irreducible polynomial distinct from $X-1$. We have the following (cf Fong-Srinivasan [5], Proposition (1A))

Proposition 2.2. Suppose that $h$ is a semisimple primary element of some general linear group $G L(m, q)$, and that $h \in\left(f^{\nu}\right)$ for some $f \neq X-1$. Writing $d$ the degree of $f$, we thus have $m=k d$, where $\nu=(1, \ldots, 1) \vdash k$. Then

$$
C_{G L(k d, q)}(h) \cong G L\left(k, q^{d}\right) .
$$

From the primary decomposition of $g \in G$, we deduce the following: if $\mathcal{F}$ is the disjoint union of $\mathcal{F}_{1}$ and $\mathcal{F}_{2}$, then there is a unique decomposition $V=V_{1} \oplus V_{2}$ where $V_{1}$ and $V_{2}$ are $g$-stable, $\left.g\right|_{V_{1}} \in\left(f^{\nu(f)}\right)_{f \in \mathcal{F}_{1}}$ and $\left.g\right|_{V_{2}} \in\left(f^{\nu(f)}\right)_{f \in \mathcal{F}_{2}}$. Then $g$ has a unique decomposition $g=g_{1} g_{2}=g_{2} g_{1}$ where $g_{1}, g_{2} \in G L(V),\left.g_{1}\right|_{V_{2}}=1$ and $\left.g_{2}\right|_{V_{1}}=1$. Indeed, under these hypotheses, necessarily, for $i \in\{1,2\}, V_{i}$ is $g_{i}$-stable and $\left.g_{i}\right|_{V_{i}}=\left.g\right|_{V_{i}}$. We then have $C_{G}(g) \cong C_{G L\left(V_{1}\right)}\left(\left.g\right|_{V_{1}}\right) \times C_{G L\left(V_{2}\right)}\left(\left.g\right|_{V_{2}}\right)$.

\section{Sections, blocks}

\subsection{Sections}

The idea of the following definitions is, in the rational canonical form we gave above, to isolate blocks corresponding to irreducible polynomials whose degree is divisible by a given (positive) integer $d$. We let, writing $\delta(f)$ the degree of any polynomial $f$,

$$
\mathcal{F}_{d}=\{f \in \mathcal{F} \mid f \neq X-1 \text { and } d \mid \delta(f)\} \text { and } \mathcal{F}_{d}^{0}=\mathcal{F} \backslash \mathcal{F}_{d} .
$$

We define the following union of conjugacy classes of $G$ :

$$
\mathcal{X}_{d}=\left\{g \in\left(f^{\nu(f)}\right)_{f \in \mathcal{F}_{d} \cup\{X-1\}} \mid \nu(X-1)=(0) \text { or }(1, \ldots, 1)\right\} .
$$


Note that we have $1 \in \mathcal{X}_{d}$.

For $x \in \mathcal{X}_{d}$, we let $\mathcal{F}_{x}=\mathcal{F}_{d}$ and $\mathcal{F}_{x}^{0}=\mathcal{F}_{d}^{0}$. Then, for each $x \in \mathcal{X}_{d}$, the set $\mathcal{F}$ is the disjoint union of $\mathcal{F}_{x}$ and $\mathcal{F}_{x}^{0}$. For each $x \in \mathcal{X}_{d}$, there exists a unique decomposition $V=V_{x} \oplus V_{x}^{0}$ such that $V_{x}$ is $x$-stable, $\left.x\right|_{V_{x}^{0}}=1$ and $\left.x\right|_{V_{x}} \in\left(f^{\nu(f)}\right)_{f \in \mathcal{F}_{x}}$. We then have $C_{G}(x) \cong C_{G L\left(V_{x}\right)}\left(\left.x\right|_{V_{x}}\right) \times G L\left(V_{x}^{0}\right)$, and we write $C_{G}(x)=H_{x} \times H_{x}^{0}$. We let

$$
\mathcal{Y}_{d}(x)=\left\{y \in G L(V) \mid V_{x}^{0} \text { is } y \text {-stable, }\left.y\right|_{V_{x}}=1 \text { and }\left.y\right|_{V_{x}^{0}} \in\left(f^{\nu(f)}\right)_{f \in \mathcal{F}_{x}^{0}}\right\} .
$$

From the definitions, we see that $\mathcal{Y}_{d}(x) \subset C_{G}(x)$ for any $x \in \mathcal{X}_{d}$. We also see, using the remarks we made on the primary decomposition, that, for any $g \in G$, there exist unique $x \in \mathcal{X}_{d}$ and $y \in \mathcal{Y}_{d}(x)$ such that $g=x y$. Indeed, if $g \in\left(f_{1}^{\nu_{1}}, \ldots, f_{r}^{\nu_{r}}\right)$ and if we write, as above, $V=V_{1} \oplus \cdots \oplus V_{r}$ and $g=g_{1} \ldots g_{r}$, then we necessarily have $V_{x}=\oplus_{i \in I} V_{i}$, where $I \subset\{1, \ldots, r\}$ is the set of indices $i$ such that $f_{i}^{\nu_{i}}$ has the property defining $\mathcal{X}_{d}, V_{x}^{0}=\oplus_{i \notin I} V_{i},\left.x\right|_{V_{x}}=\left.g\right|_{V_{x}}$ and $\left.y\right|_{V_{x}^{0}}=\left.g\right|_{V_{x}^{0}}$ (and $x \in \mathcal{X}_{d}$ and $y \in \mathcal{Y}_{d}(x)$ are uniquely determined by these conditions). We have $x=\prod_{i \in I} g_{i}$ and $y=\prod_{i \notin I} g_{i}$.

These definitions allow us to define $\left(\mathcal{X}_{d}, \mathcal{Y}_{d}\right)$-sections as introduced in section 2 of 8 . The following proposition shows that $\mathcal{X}_{d}$ and $\mathcal{Y}_{d}(x)\left(x \in \mathcal{X}_{d}\right)$ behave in many respects like the set of $r$-elements ( $r$ a prime) and the sets of $r$-regular elements of their centralizers respectively.

Proposition 3.1. Take any integer $d>0$. Then, for any $x \in \mathcal{X}_{d}$,

(i) $\mathcal{Y}_{d}(x)$ is a union of conjugacy classes of $C_{G}(x)$.

(ii) For all $y \in \mathcal{Y}_{d}(x), C_{G}(x y) \leq C_{G}(x)$.

(iii) For all $g \in G, \mathcal{Y}_{d}\left(x^{g}\right)=\mathcal{Y}_{d}(x)^{g}$.

(iv) Two elements of $x \mathcal{Y}_{d}(x)$ are $G$-conjugate if and only if they are $C_{G}(x)$ conjugate.

(v) $G=\coprod_{x \in \mathcal{X}_{d} / G}\left\{(x y)^{G}, y \in \mathcal{Y}_{d}(x) / C_{G}(x)\right\}$.

Proof. (i) We have $C_{G}(x)=H_{x} \times H_{x}^{0}$, and we see $C_{G}(x)$ as a subgroup of $G L\left(V_{x}\right) \times G L\left(V_{x}^{0}\right)$. For $y \in \mathcal{Y}_{d}(x)$, we have $y=\left(\left.y\right|_{V_{x}},\left.y\right|_{V_{x}^{0}}\right)=\left(1, y_{x}^{0}\right)$. Then, for all $h=\left(h_{x}, h_{x}^{0}\right) \in C_{G}(x), h^{-1} y h=\left(1,\left(h_{x}^{0}\right)^{-1} y_{x}^{0} h_{x}^{0}\right) \in \mathcal{Y}_{d}(x)$.

(ii) For all $y \in \mathcal{Y}_{d}(x)$, we have $C_{G}(x y) \cong C_{G L\left(V_{x}\right)}\left(\left.x\right|_{V_{x}}\right) \times C_{G L\left(V_{x}^{0}\right)}\left(\left.y\right|_{V_{x}^{0}}\right) \leq$ $C_{G L\left(V_{x}\right)}\left(\left.x\right|_{V_{x}}\right) \times G L\left(V_{x}^{0}\right) \cong C_{G}(x)$, and $C_{G}(x y) \leq C_{G}(x)$ since the isomorphism on the left is the restriction to $C_{G L\left(V_{x}\right)}\left(\left.x\right|_{V_{x}}\right) \times C_{G L\left(V_{x}^{0}\right)}\left(\left.y\right|_{V_{x}^{0}}\right)$ of the isomorphism on the right.

(iii) Take $g \in G$ and $y \in \mathcal{Y}_{d}(x)$. Then $x^{g} \in \mathcal{X}_{d}$ and $\mathcal{F}_{x^{g}}=\mathcal{F}_{x}$ (and thus $\left.\mathcal{F}_{x^{g}}^{0}=\mathcal{F}_{x}^{0}\right)$. We have $V=g^{-1} V=g^{-1} V_{x} \oplus g^{-1} V_{x}^{0}$. Furthermore, $\left.x^{g}\right|_{g^{-1} V_{x}^{0}}=1$, $g^{-1} V_{x}$ is $x^{g}$-stable, and $\left.x^{g}\right|_{g^{-1} V_{x}} \in\left(f^{\nu}\right)_{f \in \mathcal{F}_{x}}$. Thus $g^{-1} V_{x}=V_{x^{g}}$ and $g^{-1} V_{x}^{0}=$ $V_{x^{g}}^{0}$. Now, since $y \in \mathcal{Y}_{d}(x)$, we see that $\left.y^{g}\right|_{g^{-1} V_{x}}=1, g^{-1} V_{x}^{0}$ is $y^{g}$-stable, and $\left.y^{g}\right|_{g^{-1} V_{x}^{0}} \in\left(f^{\nu}\right)_{f \in \mathcal{F}_{x}^{0}}$. Hence $y^{g} \in \mathcal{Y}\left(x^{g}\right)$, and $\mathcal{Y}_{d}(x)^{g} \subset \mathcal{Y}_{d}\left(x^{g}\right)$ for all $g \in G$. Then, for any $g \in G, \mathcal{Y}_{d}\left(x^{g}\right)^{g^{-1}} \subset \mathcal{Y}_{d}(x)$ so that $\mathcal{Y}_{d}\left(x^{g}\right) \subset \mathcal{Y}_{d}(x)^{g}$. Hence the result.

(iv) Suppose that, for some $y, z \in \mathcal{Y}_{d}(x)$, there exists $h \in G$ such that $x y=$ $h^{-1} x z h$. Writing $g=x y$, we also have $g=x^{h} z^{h}$, and $x^{h} \in \mathcal{X}_{d}$ (since $\mathcal{X}_{d}$ is a union of $G$-conjugacy classes) and $z^{h} \in \mathcal{Y}_{d}\left(x^{h}\right)$ (by (iii)). By the uniqueness of such a writing for $g$, we have $x^{h}=x$ and $z^{h}=y$. Hence $h \in C_{G}(x)$. In particular, $x y$ and $x z$ are $C_{G}(x)$-conjugate. 
(v) For any $g \in G$, there exists a unique $x \in \mathcal{X}$ such that $g \in x \mathcal{Y}_{d}(x)$. Thus

$$
\begin{aligned}
G & =\coprod_{x \in \mathcal{X}_{d}} x \mathcal{Y}_{d}(x) \\
& =\coprod_{x \in \mathcal{X}_{d} / G}\left(x \mathcal{Y}_{d}(x)\right)^{G} \text { (because of (iii)) } \\
& =\coprod_{x \in \mathcal{X} / G} \bigcup_{y \in \mathcal{Y}_{d}(x)}(x y)^{G} \\
& =\coprod_{x \in \mathcal{X}_{d} / G} \coprod_{y \in \mathcal{Y}_{d}(x) / C_{G}(x)}(x y)^{G},
\end{aligned}
$$

this last equality being true by (iv) (if $(x y)^{g}=(x z)^{g^{\prime}}$, then $x y=x z^{g^{\prime} g^{-1}}$ so that, by (iv), $y$ and $z$ are $C_{G}(x)$-conjugate).

Because of the analogy we mentioned before the proposition, we introduce some terminology. We will refer to the elements of $\mathcal{X}_{d}$ as d-elements. One set of particular importance is $\mathcal{Y}_{d}(1)$. This is the set of elements of $G$ whose minimal polynomial has no irreducible factor of degree divisible by $d$ (except possibly $X-1)$. We call the elements of $\mathcal{Y}_{d}(1)$ the $d$-regular elements of $G$. The elements of $G \backslash \mathcal{Y}_{d}(1)$ are called $d$-singular.

Note that, with these definitions, a 1-regular element of $G$ is an element whose minimal polynomial has no irreducible factor of degree divisible by 1 , except $X-1$. This means that its minimal polynomial is a power of $X-1$. Hence the 1-regular elements of $G L(n, q)$ are precisely the unipotent elements.

For any $x \in \mathcal{X}_{d}$, we call the union of the $G$-conjugacy classes meeting $x \mathcal{Y}_{d}(x)$ the $\mathcal{Y}_{d}$-section of $x$. The above proposition implies that, for each $x \in \mathcal{X}_{d}$, induction of complex class functions gives an isometry from the space of class functions of $C_{G}(x)$ vanishing outside $x \mathcal{Y}_{d}(x)$ onto the space of class functions of $G$ vanishing outside the $\mathcal{Y}_{d}$-section of $x$ (cf [8]).

We remark that the $\mathcal{Y}_{d}$-sections of $G$ are quite different from ordinary prime sections. Take any $1 \neq x \in \mathcal{X}_{d}$. Then, by definition, $\mathcal{Y}_{d}(x) \subset \mathcal{Y}_{d}(1) \cap C_{G}(x)$. However, if $d \neq 1$, then there exists $\lambda \in \mathbf{F}_{q}^{\times}$such that $\lambda I_{n} \in \mathcal{Y}_{d}(1) \cap C_{G}(x)$ but $\lambda I_{n} \notin \mathcal{Y}_{d}(x)$, so that $\mathcal{Y}_{d}(x) \neq \mathcal{Y}_{d}(1) \cap C_{G}(x)$ (while this equality holds when we define $\left(\mathcal{X}_{d}, \mathcal{Y}_{d}\right)$-sections to be the ordinary $r$-sections for some prime $r$ ).

Furthermore, still supposing $d \neq 1$, if $x$ is a (non trivial) $r$-element of $G$ for some prime $r$, then, most of the time (that is, when $q-1$ is not a power of $r)$, there exists an $r$-regular element $\lambda \in \mathbf{F}_{q}^{\times}$such that $\lambda I_{n} \notin \mathcal{Y}_{d}(x)$, so that $x \lambda I_{n} \notin(x \mathcal{Y}(x))^{G}$. But $x \lambda I_{n}$ belongs to the $r$-section of $x$. Hence the $\mathcal{Y}_{d}$-section of $x$ is not a union of $r$-sections.

\subsection{Blocks}

With the definition of $d$-regular element we have given, we can now define generalized blocks of irreducible characters, as described in the introduction, by taking $\mathcal{C}$ to be the set $\mathcal{Y}_{d}(1)$ of $d$-regular elements. For any positive integer 
$d$, the $d$-blocks of $G$ are defined to be minimal subsets of $\operatorname{Irr}(G)$ which are orthogonal across $d$-regular elements. Recall that two characters $\chi, \psi \in \operatorname{Irr}(G)$ are orthogonal across $d$-regular elements if

$$
<\chi, \psi>_{\mathcal{Y}_{d}(1)}:=\frac{1}{|G|} \sum_{y \in \mathcal{Y}_{d}(1)} \chi(y) \overline{\psi(y)}=0 .
$$

Otherwise, $\chi$ and $\psi$ are said to be directly linked across $d$-regular elements.

We will particularly consider the restriction of the relation of $\mathcal{Y}_{d}(1)$-linking to the subset of unipotent characters. The unipotent characters of $G$ are the irreducible components of the permutation representation of $G$ on the cosets of a Borel subgroup (i.e. the normalizer in $G$ of a Sylow $p$-subgroup of $G$, where $p$ is the defining characteristic). They are labeled by the partitions of $n$. For $\lambda \vdash n$, we will write $\chi_{\lambda}$ the unipotent (irreducible) character of $G$ labeled by $\lambda$. We write $\operatorname{Unip}(G)=\left\{\chi_{\lambda}, \lambda \vdash n\right\}$. The equivalence classes of $\operatorname{Unip}(G)$ modulo the restriction of $\mathcal{Y}_{d}(1)$-linking will be called unipotent $d$-blocks of $G$. It is clear that, for any $d$-block $B$ of $G, B \cap \operatorname{Unip}(G)$ is a union of unipotent $d$-blocks.

\section{Nakayama Conjecture for unipotent blocks}

\subsection{Murnaghan-Nakayama Rule for unipotent characters}

Pick $g \in G$, and write $\operatorname{Char}(g)=\prod_{i} f_{i}^{k_{i}}$ and the corresponding decomposition $g=\prod_{i} g_{i}$. Then pick $i_{0}$, and write $g=\rho \sigma$, where

$$
\rho=g_{i_{0}} \text { and } \sigma=\prod_{i \neq i_{0}} g_{i} .
$$

Now fix some matrix representation of $G$, and see $G$ as $G L(n, q)$. Writing $d$ the degree of $f_{i_{0}}, m=k_{i_{0}} d$, and $l=n-m$, we have, writing $\sim$ for equivalence of matrices over $\mathbf{F}_{q}$,

$$
\begin{gathered}
g \sim\left(\begin{array}{ccc}
\ddots & & \\
& U_{\nu_{i}}\left(f_{i}\right) & \\
& & \ddots
\end{array}\right), \\
\rho \sim\left(\begin{array}{lll}
I_{l} & & \\
& U_{\nu_{i_{0}}}\left(f_{i_{0}}\right)
\end{array}\right)
\end{gathered}
$$

(and we may consider that $\rho \in G_{0}=G L(m, q)$ ), and

$$
\sigma \sim\left(\begin{array}{cccc}
() & & & \\
& \ddots & & \\
& & () & \\
& & & I_{m}
\end{array}\right)
$$

(and we may consider that $\sigma \in G_{1}=G L(l, q)$ ).

Then, using the results on the Jordan decomposition given in the first section, we see that the semi-simple part $\rho_{S}$ of $\rho$ is equivalent to

$$
\left(\begin{array}{cc}
I_{l} & \\
& U_{k_{i_{0}}}\left(f_{i_{0}}\right)
\end{array}\right) \text {. }
$$


We have $\rho_{S} \in G L(n, q)$ and $\operatorname{Char}\left(\rho_{S}\right)=f_{i_{0}}^{k_{i_{0}}}(X-1)^{l}$, and we may consider that $\rho_{S} \in G_{0}=G L(m, q)$ and $\operatorname{Char}\left(\rho_{S}\right)=f_{i_{0}}^{k_{i_{0}}}$.

We have $C_{G}\left(\rho_{S}\right)=H=H_{0} \times H_{1}$, where $H_{1} \cong G L(l, q)$ and $H_{0}=C_{G L(m, q)}\left(\rho_{S}\right) \cong G L\left(k_{i_{0}}, q^{d}\right)$.

We find in [5] an analogue of the Murnaghan-Nakayama Rule which applies to unipotent characters of $G$. More precisely, if $\chi_{\nu} \in \operatorname{Unip}(G)$, then the following theorem gives us information on the values of a class function $\chi^{\nu}$ which is, up to a sign, the same as $\chi_{\nu}$ (it is this class function, rather than the unipotent character, which appears naturally in the Deligne-Lusztig theory).

Theorem 4.1 (Murnaghan-Nakayama Rule). ([5], Theorem (2G))

Let $g \in G$, and $\rho$ and $\sigma$ be as above, and let $\nu \vdash n$. Then

$$
\chi^{\nu}(\rho \sigma)=\sum_{\lambda \in \mathcal{L}_{\nu}} a_{\nu \lambda}^{\rho} \chi^{\lambda}(\sigma),
$$

where $\mathcal{L}_{\nu}$ is the set of partitions $\lambda$ of $l$ which can be obtained from $\nu$ by removing $k_{i_{0}} d$-hooks, and $a_{\nu \lambda}^{\rho} \in \mathbf{Z}\left[q^{d}\right]$.

If $\mathcal{L}_{\nu}=\emptyset$, then $\chi^{\nu}(\rho \sigma)=0$.

We have $a_{\nu \lambda}^{\rho} \neq 0$ for $\lambda \in \mathcal{L}_{\nu}$.

(The coefficients of $a_{\nu \lambda}^{\rho}$ depend on the characters of the symmetric group $S_{k_{i_{0}}}$ and the Green functions of $G L\left(k_{i_{0}}, q^{d}\right) \cong H_{0}$ (applied to the unipotent part $\rho_{U}$ of $\rho$ ), and all the non-zero coefficients of $a_{\nu \lambda}^{\rho}$ have the same sign).

Remark: it is easy to see that, if $a_{\nu \lambda}^{\rho} \neq 0$, then $\nu$ and $\lambda$ have the same $d$-core.

The idea is to use this theorem recursively so as to be able to obtain information about $<\chi^{\lambda}, \chi^{\mu}>_{x \mathcal{Y}_{d}(x)}$, for $\lambda, \mu \vdash n$ and $x \in \mathcal{X}_{d}$. We first use it to obtain a formula for $\chi^{\mu}(x y)$, where $\mu \vdash n$ and $y \in \mathcal{Y}_{d}(x)$. We take $1 \neq x \in \mathcal{X}_{d}$. Suppose $x \in c_{x}=\left(f_{1}^{\lambda_{1}}, \ldots, f_{s}^{\lambda_{s}}\right)$ where, for each $1 \leq i \leq s, \lambda_{i} \vdash k_{i}$, and $d \mid \delta\left(f_{i}\right)$ or $f_{i}=X-1$. If $f_{i} \neq X-1$, we let $\delta\left(f_{i}\right)=m_{i} d$. From the definition of $\mathcal{X}_{d}$, we see that, in the primary decomposition of $x$, we may omit the component corresponding to $X-1$, because it is necessarily the identity. We relabel $f_{1}, \ldots, f_{r}$ the $f_{i}$ 's which are distinct from $X-1$, and we write $x=x_{1} \ldots x_{r}$, where each $x_{i}$ has exactly one elementary divisor distinct from $X-1$ (namely, $f_{i}$ ), and with the same multiplicity as in $\operatorname{Char}(x)$. We will say that $x$ has $d$-type $\left(k_{1} m_{1}, \ldots, k_{r} m_{r}\right)$.

By repeated use of the Murnaghan-Nakayama Rule, we obtain that, for any $y \in \mathcal{Y}_{d}(x)$ and for $\mu \vdash n$, we have

$$
\begin{aligned}
\chi^{\mu}(x y) & =\sum_{\mu_{1} \in \mathcal{L}_{\mu}} a_{\mu \mu_{1}}^{x_{1}} \chi^{\mu_{1}}\left(x_{2} \ldots x_{r} y\right) \\
& =\sum_{\mu_{1} \in \mathcal{L}_{\mu}} a_{\mu \mu_{1}}^{x_{1}}\left(\sum_{\mu_{2} \in \mathcal{L}_{\mu_{1}}} a_{\mu_{1} \mu_{2}}^{x_{2}} \chi^{\mu_{2}}\left(x_{3} \ldots x_{r} y\right)\right), \\
& =\sum_{\mu_{1} \in \mathcal{L}_{\mu}} \cdots \sum_{\mu_{r} \in \mathcal{L}_{\mu_{r-1}}} a_{\mu \mu_{1}}^{x_{1}} \ldots a_{\mu_{r-1} \mu_{r}}^{x_{r}} \chi^{\mu_{r}}(y)
\end{aligned}
$$

which can be written

$$
\chi^{\mu}(x y)=\sum_{\lambda \in \mathcal{L}_{\left(k_{1} m_{1}, \ldots, k_{r} m_{r}\right) d}^{\mu}} \alpha_{\mu \lambda}^{\left(x_{1}, \ldots, x_{r}\right)} \chi^{\lambda}(y),
$$


where the $\alpha_{\mu \lambda}^{\left(x_{1}, \ldots, x_{r}\right)}$ 's are integers and $\mathcal{L}_{\left(k_{1} m_{1}, \ldots, k_{r} m_{r}\right) d}^{\mu}$ is the set of partitions of $n-\left(\sum_{i} k_{i} m_{i}\right) d$ which can be obtained from $\mu$ by removing $k_{1}\left(m_{1} d\right)$-hooks, then $k_{2}\left(m_{2} d\right)$-hooks, $\ldots$, and finally $k_{r}\left(m_{r} d\right)$-hooks. We will call such a sequence of removals a $\left(k_{1} m_{1}, \ldots, k_{r} m_{r}\right) d$-path from $\mu$ to $\lambda$.

Note that, in this sum, each $\lambda$ can appear several times, if there is more than one $\left(k_{1} m_{1}, \ldots, k_{r} m_{r}\right) d$-path from $\mu$ to $\lambda$. Note also that, in the right side of this equality, $y$ has implicitely been seen as an element of $G L(l, q)$, where $l=n-\left(\sum_{i} k_{i} m_{i}\right) d$.

If $\mathcal{L}_{\left(k_{1} m_{1}, \ldots, k_{r} m_{r}\right) d}^{\mu}=\emptyset$, then $\chi^{\mu}(x y)=0$.

We have $\alpha_{\mu \lambda}^{\left(x_{1}, \ldots, x_{r}\right)} \in \mathbf{Z}\left[q^{d}\right]$, and, if we separate the possibly multiple occurences

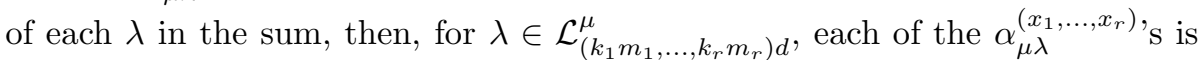
non-zero (these are indexed by the $\left(k_{1} m_{1}, \ldots, k_{r} m_{r}\right) d$-paths from $\mu$ to $\lambda$ ).

If $\alpha_{\mu \lambda}^{\left(x_{1}, \ldots, x_{r}\right)} \neq 0$, then, since there is a $\left(k_{1} m_{1}, \ldots, k_{r} m_{r}\right) d$-path from $\mu$ to $\lambda$, and since the removal of a hook of length $m d$ can be obtained by the removal of a sequence of $m$ hooks of length $d$, we see that $\mu$ and $\lambda$ have the same $d$-core.

We call the $\alpha_{\mu \lambda}^{\left(x_{1}, \ldots, x_{r}\right)}$,s the $M N$-coefficients, and we will from now on write $\alpha_{\mu \lambda}^{x}$ for $\alpha_{\mu \lambda}^{\left(x_{1}, \ldots, x_{r}\right)}$.

\subsection{One direction of the Nakayama Conjecture}

We are now able to prove that the unipotent $d$-blocks of $G$ satisfy one direction of a generalized Nakayama Conjecture. The proof we give is an adaptation to our case of the proof given by Külshammer, Olsson and Robinson in the case of symmetric groups.

Take $x \in \mathcal{X}_{d}$ of $d$-type $\mathbf{k m}=\left(k_{1} m_{1}, \ldots, k_{r} m_{r}\right)$. We let $l=n-\mathbf{k m} d=$ $l-\left(\sum_{i=1}^{r} k_{i} m_{i}\right) d$. Writing $G_{0}=G L(\mathbf{k m} d, q)=\prod_{i=1}^{r} G L\left(k_{i} m_{i} d, q\right)$ and $G_{1}=$ $G L(l, q)$, we have $x=\left(x_{0}, x_{1}\right)=\left(x_{0}, 1\right) \in G_{0} \times G_{1}$. Then $C_{G}(x)=H_{0} \times H_{1}$, where $H_{1} \cong G L(l, q)$ and $H_{0}=C_{G_{0}}\left(x_{0}\right)$ (note that, if $x$ is semisimple, then $\left.H_{0} \cong G L\left(\mathbf{k}, q^{\mathbf{m} d}\right) \cong \prod_{i=1}^{r} G L\left(k_{i}, q^{m_{i} d}\right)\right)$.

Now take $y \in \mathcal{Y}_{d}(x)$. Then, as an element of $C_{G}(x)=H_{0} \times H_{1}$, we have $y=\left(y_{0}, y_{1}\right)=\left(1, y_{1}\right)$.

Writing $\mathcal{Y}_{d}^{t}(u)$ for $\mathcal{Y}_{d}(u)$ when $u \in G L(t, q)$, we have that $y$, element of $C_{G}(x)$, belongs to $\mathcal{Y}_{d}^{n}(x)$ if and only if $y=\left(1, y_{1}\right) \in H_{0} \times H_{1}$, where $y_{1}$ belongs to $\mathcal{Y}_{d}^{l}(1)$. Hence $\mathcal{Y}_{d}^{n}(x)$ is in natural one to one correspondence with $\mathcal{Y}_{d}^{l}(1)$. Now we consider $\mu, \mu^{\prime} \vdash n$, and $x \in \mathcal{X}_{d}$ of $d$-type $\mathbf{k m}=\left(k_{1} m_{1}, \ldots, k_{r} m_{r}\right)$. We have

$$
\begin{aligned}
& <\chi^{\mu}, \chi^{\mu^{\prime}}>_{x \mathcal{Y}_{d}(x)}=\frac{1}{|G|} \sum_{y \in \mathcal{Y}_{d}(x)} \chi^{\mu}(x y) \overline{\chi^{\mu^{\prime}}(x y)} \\
& =\frac{1}{|G|} \sum_{y=\left(y_{0}, y_{1}\right) \in \mathcal{Y}_{d}(x)}\left[\left(\sum_{\lambda \in \mathcal{L}_{\mathbf{k m}}^{\mu}} \alpha_{\mu \lambda}^{x} \chi^{\lambda}\left(y_{1}\right)\right)\left(\sum_{\lambda^{\prime} \in \mathcal{L}_{\mathbf{k m}}^{\mu^{\prime}}} \alpha_{\mu^{\prime} \lambda^{\prime}}^{x} \overline{\chi^{\lambda^{\prime}}\left(y_{1}\right)}\right)\right] \\
& =\frac{1}{|G|} \sum_{y_{1} \in \mathcal{Y}_{d}^{l}(1)} \sum_{\lambda \in \mathcal{L}_{\mathbf{k m}}^{\mu}, \lambda^{\prime} \in \mathcal{L}_{\mathbf{k m}}^{\mu^{\prime}}} \alpha_{\mu \lambda}^{x} \alpha_{\mu^{\prime} \lambda^{\prime}}^{x} \chi^{\lambda}\left(y_{1}\right) \overline{\chi^{\lambda^{\prime}}\left(y_{1}\right)}
\end{aligned}
$$

(by the above remark on $\mathcal{Y}_{d}(x)$ ). 
We write $A_{\mu \mu^{\prime}}^{x}=<\chi^{\mu}, \chi^{\mu^{\prime}}>_{x} \mathcal{Y}(x)$. Then

$$
\begin{aligned}
& A_{\mu \mu^{\prime}}^{x}=\frac{1}{|G|} \sum_{\lambda \in \mathcal{L}_{\mathrm{km}}^{\mu}, \lambda^{\prime} \in \mathcal{L}_{\mathrm{km}}^{\mu^{\prime}}} \alpha_{\mu \lambda}^{x} \alpha_{\mu^{\prime} \lambda^{\prime}}^{x} \sum_{y_{1} \in \mathcal{Y}_{d}^{l}(1)} \chi^{\lambda}\left(y_{1}\right) \overline{\chi^{\lambda^{\prime}}\left(y_{1}\right)} \\
& =\frac{\left|H_{1}\right|}{|G|} \sum_{\lambda \in \mathcal{L}_{\mathbf{k m}}^{\mu}, \lambda^{\prime} \in \mathcal{L}_{\mathbf{k m}}^{\mu^{\prime}}} \alpha_{\mu \lambda}^{x} \alpha_{\mu^{\prime} \lambda^{\prime}}^{x}<\chi^{\lambda} \chi^{\lambda^{\prime}}>_{\mathcal{Y}^{l}}^{l}(1)
\end{aligned}
$$

i.e.

$$
A_{\mu \mu^{\prime}}^{x}=\frac{\left|H_{1}\right|}{|G|} \sum_{\lambda \in \mathcal{L}_{\mathbf{k m}}^{\mu}, \lambda^{\prime} \in \mathcal{L}_{\mathbf{k m}}^{\mu^{\prime}}} \alpha_{\mu \lambda}^{x} \alpha_{\mu^{\prime} \lambda^{\prime}}^{x} A_{\lambda \lambda^{\prime}}^{1} .
$$

We use induction on $n$ to prove that, if $A_{\mu \mu^{\prime}}^{x} \neq 0$, then $\mu$ and $\mu^{\prime}$ have the same $d$-core. We may assume that $\mu \neq \mu^{\prime}$.

If $n<d$, then each partition is its own $d$-core. Furthermore, in this case, $\mathcal{X}_{d}=\{1\}$ and $\mathcal{Y}_{d}(1)=G$. Thus, for all $x \in \mathcal{X}_{d}$, we have $A_{\mu \mu^{\prime}}^{x}=A_{\mu \mu^{\prime}}^{1}$, and $A_{\mu \mu^{\prime}}^{1}=<\chi^{\mu}, \chi^{\mu^{\prime}}>_{G}=0\left(\right.$ since $\left.\mu \neq \mu^{\prime}\right)$. Hence the result is true in this case. Thus, we suppose $n \geq d$. First suppose $x \neq 1$ and $A_{\mu \mu^{\prime}}^{x} \neq 0$. Then

$$
A_{\mu \mu^{\prime}}^{x}=\frac{\left|H_{1}\right|}{|G|} \sum_{\lambda \in \mathcal{L}_{\mathbf{k m}}^{\mu}, \lambda^{\prime} \in \mathcal{L}_{\mathbf{k m}}^{\mu^{\prime}}} \alpha_{\mu \lambda}^{x} \alpha_{\mu^{\prime} \lambda^{\prime}}^{x} A_{\lambda \lambda^{\prime}}^{1} \neq 0 .
$$

Thus there exist $\lambda \in \mathcal{L}_{\mathbf{k m}}^{\mu}$ and $\lambda^{\prime} \in \mathcal{L}_{\mathbf{k m}}^{\mu^{\prime}}$ such that $\alpha_{\mu \lambda}^{x} \alpha_{\mu^{\prime} \lambda^{\prime}}^{x} A_{\lambda \lambda^{\prime}}^{1} \neq 0$. Then $\alpha_{\mu \lambda}^{x} \neq 0$ implies that $\mu$ and $\lambda$ have the same $d$-core, and $\alpha_{\mu^{\prime} \lambda^{\prime}}^{x \neq 0}$ implies that $\mu^{\prime}$ and $\lambda^{\prime}$ have the same $d$-core. And, by the induction hypothesis (applied to $n-\mathbf{k m} d<n), A_{\lambda \lambda^{\prime}}^{1} \neq 0$ implies that $\lambda$ and $\lambda^{\prime}$ have the same $d$-core.

Now, if $x=1$, we see, by the existence and uniqueness of the decomposition we introduced, that

$$
0=<\chi^{\mu}, \chi^{\mu^{\prime}}>_{G}=\sum_{x \in \mathcal{X}_{d}}<\chi^{\mu}, \chi^{\mu^{\prime}}>_{x \mathcal{Y}_{d}(x)}=\sum_{x \in \mathcal{X}_{d}} A_{\mu \mu^{\prime}}^{x} .
$$

Hence, if $A_{\mu \mu^{\prime}}^{1} \neq 0$, then there exists an $x^{\prime} \in \mathcal{X}_{d} \backslash\{1\}$ such that $A_{\mu \mu^{\prime}}^{x^{\prime}} \neq 0$. This in turn implies, by the previous case, that $\mu$ and $\mu^{\prime}$ have the same $d$-core. Skipping back from class functions to irreducible characters, we see that we have proved the following

Theorem 4.2. Let $d>0$ be any integer. If two unipotent (irreducible) characters $\chi_{\mu}$ and $\chi_{\mu^{\prime}}$ of $G=G L(V)$ are directly linked across some $x \mathcal{Y}_{d}(x)$, where $x \in \mathcal{X}_{d}$, then $\mu$ and $\mu^{\prime}$ have the same $d$-core (and this is true in particular for $x=1)$.

Extending by transitivity the relation of direct $\mathcal{Y}_{d}(1)$-linking, we obtain

Theorem 4.3. If two unipotent characters $\chi_{\mu}$ and $\chi_{\mu^{\prime}}$ of $G$ are in the same unipotent $d$-block of $G$, then $\mu$ and $\mu^{\prime}$ have the same $d$-core.

Each unipotent $d$-block of $G$ is therefore associated to a $d$-core. For each given $d$-core $\gamma$, we can consider the union of the (possibly many) unipotent $d$ blocks associated to $\gamma$. The (a priori) bigger blocks obtained in this way are parametrized by the set of $d$-cores of partitions of $n$, and they satisfy an analogue 
of the Nakayama Conjecture. In accordance with the terminology used in 8 , we call them combinatorial unipotent d-blocks.

We would like to prove that, in fact, the notions of unipotent $d$-block and combinatorial unipotent $d$-block coincide. Unfortunately, it seems hard to prove (of infirm) in general. In the following sections, we give examples where the computations are easier, so that the result can be established.

\subsection{The case $d=1$}

In this section, we consider the extreme case $d=1$. Recall that the 1-regular elements of $G=G L(V)$ are just the unipotent elements. Note that all partitions of $n$ have the same 1-core (the only 1-core there is, that is the empty one). In order to prove an analogue of the Nakayama Conjecture for unipotent 1-blocks, we therefore need to prove that any two unipotent characters belong to the same 1-block. We actually prove more than that. Namely, any irreducible character of $G$ is directly linked across unipotent elements to the trivial character.

Our tool to deal with this case is the Alvis-Curtis duality. D. Alvis has proved (cf [1]) that there is an isometry of order $2, D_{G}: \operatorname{ch}(G) \longrightarrow \operatorname{ch}(G)$, where $\operatorname{ch}(G)$ denotes the ring of virtual characters of $G$ (in fact, this is true in a much more general context, that is when $G$ is any finite group with a split $(B, N)$-pair of characteristic $p)$. In particular, the image under the duality map $D_{G}$ of any irreducible (complex) character of $G$ is, up to a sign, an irreducible character. The case of unipotent characters has been made more precise by C. W. Curtis (cf [2]). Namely, if $\chi_{\lambda} \in \operatorname{Unip}(G)$, then $D_{G}\left(\chi_{\lambda}\right)=\chi_{\varepsilon \lambda}$, where $\varepsilon$ is the sign character of the symmetric group $S_{n}$ (that is, $\chi_{\varepsilon \lambda}$ is the unipotent character $\chi_{\mu}$ where $\varphi_{\mu}$ is the irreducible character of $S_{n}$ such that $\left.\varphi_{\mu}=\varepsilon \varphi_{\lambda}\right)$. This can be written as $D_{G}\left(\chi_{\lambda}\right)=\chi_{\lambda^{*}}$, where $\lambda^{*}$ is the partition conjugate to $\lambda$. In particular, we have $D_{G}\left(1_{G}\right)=\chi_{\varepsilon}=S t_{G}$, the Steinberg character of $G$.

We define the virtual character $\chi_{u}$ of $G$ via

$$
\chi_{u}(g)=\left\{\begin{array}{l}
1 \text { if } g \text { is unipotent } \\
0 \text { otherwise }
\end{array} .\right.
$$

We then have the following:

Lemma 4.4. ([3], Lemme 11.1) We have $\chi_{u}=D_{G}\left(|G|_{p^{\prime}}^{-1} r e g_{G}\right)$, where reg $g_{G}$ is the character of the regular representation of $G$.

We let $G_{u}$ be the set of unipotent elements of $G$. Then, for any $\chi \in \operatorname{Irr}(G)$, we have

$$
<\chi, 1_{G}>_{G_{u}}:=\frac{1}{|G|} \sum_{g \in G_{u}} \chi(g)=\frac{1}{|G|} \sum_{g \in G} \chi(g) \chi_{u}(g)=<\chi, \chi_{u}>_{G} .
$$

Thus, since $D_{G}$ is an isometry, we have

$<\chi, 1_{G}>_{G_{u}}=<D_{G}(\chi), D_{G}\left(\chi_{u}\right)>_{G}=\frac{1}{|G|_{p^{\prime}}}<D_{G}(\chi), r e g_{G}>_{G}=\frac{1}{|G|_{p^{\prime}}} D_{G}(\chi)(1)$.

Hence $<\chi, 1_{G}>_{G_{u}} \neq 0$, i.e. $\chi$ and $1_{G}$ are directly linked across unipotent elements. We thus have 
Theorem 4.5. The only 1-block of $G$ is $\operatorname{Irr}(G)$, and the only unipotent 1-block of $G$ is $\operatorname{Unip}(G)$. In particular, the unipotent 1-blocks of $G$ satisfy an analogue of the Nakayama Conjecture.

Note that, if $\chi=\chi_{\lambda} \in \operatorname{Unip}(G)$, then we have $<\chi_{\lambda}, 1_{G}>_{G_{u}}=\frac{1}{|G|_{p^{\prime}}} \chi_{\lambda^{*}}(1)$.

\section{$4.4 d$-weight and simple partitions}

From now on, we fix the integers $n$ and $d, d \neq 1$, and some $d$-core $\gamma$. Our aim is to prove that, if two partitions $\lambda$ and $\mu$ of $n$ have the same $d$-core $\gamma$, then the unipotent characters $\chi_{\lambda}$ and $\chi_{\mu}$ belong to the same unipotent $d$-block of $G=G L(V)$. However, if we simply write down the inner product of $\chi_{\lambda}$ and $\chi_{\mu}$ on $d$-regular (or $d$-singular) elements of $G$, we obtain a huge expression which is very hard to manipulate, let alone trying to prove that it is non-zero.

Instead, what we do in sections 4.5 and 4.6 is build, under some extra assumption on $\lambda$ and $\mu$, a chain of unipotent characters $\chi_{0}=\chi_{\lambda}, \chi_{1}, \ldots, \chi_{r}=\chi_{\mu}$, such that, for each $0 \leq i \leq r-1, \chi_{i}$ and $\chi_{i+1}$ are directly linked across $d$-singular elements. This proves that $\chi_{\lambda}$ and $\chi_{\mu}$ are linked across $d$-singular elements, and therefore belong to the same unipotent $d$-block.

Section 4.5 is devoted to the statement and proof of Theorem 4.6, which corresponds to the "elementary links" in our chain of characters. In section 4.6, we then show that, if $\lambda$ and $\mu$ have "weight small compared to $d$ ", then it is possible to build such a chain between $\chi_{\lambda}$ and $\chi_{\mu}$ (cf Theorem 4.10).

We now introduce the observations and notions we will use to construct our chain.

We can associate to each combinatorial unipotent $d$-block a $d$-weight, the $d$-weight of any partition of $n$ labeling some unipotent character in the block.

The first observation comes from the Murnaghan-Nakayama Rule: if $\lambda$ has $d$-weight $w$, then $\chi_{\lambda}$ vanishes on any element of $G$ whose $d$-part has $d$-type $\left(k_{1} m_{1}, \ldots, k_{r} m_{r}\right)$ with $\sum_{i} k_{i} m_{i}>w$.

This leads to the first definition and simplification. For any element $g \in$ $G$ with $d$-part of $d$-type $\left(k_{1} m_{1}, \ldots, k_{r} m_{r}\right)$, we define the $d$-weight of $g$ to be $\sum_{i} k_{i} m_{i}$. Thus, for partitions of $d$-weight $w$, it is enough to consider the inner product of $\chi_{\lambda}$ and $\chi_{\mu}$ on $d$-singular elements of $d$-weight at most $w$.

The Murnaghan-Nakayama Rule also implies that, if, for all $m>1, \lambda$ contains no $m d$-hook, then $\chi_{\lambda}$ vanishes on any element $g$ with $d$-part of $d$-type $\left(k_{1} m_{1}, \ldots, k_{r} m_{r}\right)$ when there exists $i$ such that $m_{i}>1$ (i.e. whenever there exists an irreducible factor of degree $m d$ with $m>1$ in $\operatorname{Min}(g))$. If $\lambda$ is such a partition, we will say that $\lambda$ is simple (or $d$-simple).

Given any partition $\lambda$ of $n$, a useful and convenient way to store the $d$ information about $\lambda$ is with an abacus. For a complete definition, we refer to 7], Section 2.7 (note however that the abacus we describe here is the horizontal mirror image of that used by James and Kerber).

We present the construction of the abacus with an example: we take $d=3$ and the partition $\lambda=(6,5,5,2,1)$ of $n=19$. We put the Young diagram of $\lambda$ in the upper-left corner of a quarter plane, and consider its (infinite) rim, in bold below. We choose an arbitrary origin, indicated by the symbol $\triangleright$, on the vertical axis. 


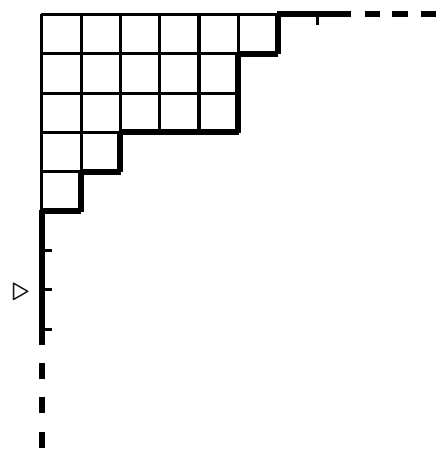

We see the rim as an infinite sequence of vertical and horizontal dashes of length 1 (the length of a box in the diagram). Writing 1 for a vertical dash and 0 for a horizontal one, we encode the rim as the following sequence, where we indicate the origin as before:

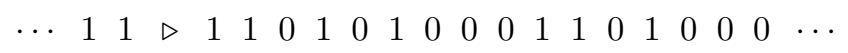

We now construct the abacus using this sequence. We put beads on $d=3$ runners, going from left to right and bottom to top, putting a bead for each 1 and an empty spot for each 0 . Here again, we indicate the origin. We get

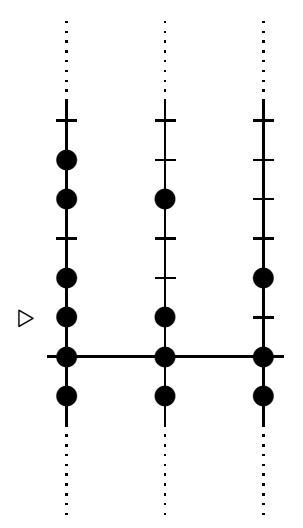

We see that, up to the choice of the runner where we put the origin, there is bijection that associates to each partition a unique abacus.

The $d$-information is visible in the abacus in a natural way. If $k$ is a positive integer, then any $k d$-rim-hook in $\lambda$ corresponds to a sequence of $k d+1$ digits in $(\dagger)$ which starts with a 0 and ends with a 1 , and to a bead in the abacus which lies, on the same runner, $k$ places above an empty spot. Moreover, these correspondences are bijective.

The removal of a $k d$-rim-hook in the Young diagram is achieved by exchanging the 0 and 1 at the extremity of the corresponding subsequence of $(\dagger)$, and by moving the corresponding bead to the empty spot.

By removing all the $d$-hooks from its Young diagram, we get the $d$-core of $\lambda$, and the corresponding sequence and abacus: 


$$
\cdots 1 \triangleright 111110100100 \cdots
$$

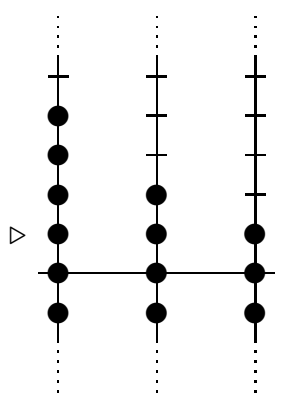

To each runner of the abacus, we associate a partition as follows. When we move all the beads as far down as possible, we get one part for each bead that we move, of length the number of places the bead goes down. The resulting $d$-tuple $\left(\lambda^{(1)}, \ldots, \lambda^{(d)}\right)$ of partitions is the $d$-quotient of $\lambda$. In the above example, we get $\left(\lambda^{(1)}, \lambda^{(2)}, \lambda^{(3)}\right)=((1,1),(2),(1))$. The lengths of the $\lambda^{(i)}$ 's add up to the $d$-weight $w$ of $\lambda$. For any positive integer $k$, we call $k$-hook in the quotient of $\lambda$ any $k$-hook in one of the $\lambda^{(i)}$ 's. Then there is a bijection between the set of $k$-hooks in the $d$-quotient of $\lambda$ and the set of $k d$-hooks in $\lambda$.

One sees easily that the partition $\lambda$ is uniquely determined by its $d$-core and $d$-quotient.

From the definition we gave above and the description of the abacus, we see that, if $\lambda$ is $d$-simple of weight $w$, then the moves corresponding to the removals of all the $d$-hooks from $\lambda$ must be stored on exactly $w$ distinct runners of the abacus (so that, in particular, one must have $w \leq d$ ), and they are all elementary, i.e. involve each a single bead which can be taken exactly once one place down.

We say that two partitions are disjoint if the possible moves on their abacus are stored on different runners. In terms of quotients, we see that two partitions $\lambda$ and $\mu$, with $d$-quotients $\left(\lambda^{(1)}, \ldots, \lambda^{(d)}\right)$ and $\left(\mu^{(1)}, \ldots, \mu^{(d)}\right)$ respectively, are disjoint if and only if, for each $1 \leq i \leq d, \lambda^{(i)} \neq \emptyset$ implies $\mu^{(i)}=\emptyset$.

For any partition $\lambda$ and integer $i \geq 0$, we write $\mathcal{L}_{\lambda}^{i}$ (respectively $\mathcal{L}_{\lambda}^{(i)}$ ) the set of partitions we can get by removing $i d$-hooks (respectively one $i d$-hook) from $\lambda$. In particular, $\mathcal{L}_{\lambda}^{0}=\{\lambda\}$, and, if $\lambda$ has $d$-weight $w$ and $d$-core $\gamma$, then $\mathcal{L}_{\lambda}^{w}=\{\gamma\}$ and, for all $i>w, \mathcal{L}_{\lambda}^{i}=\emptyset$.

\subsection{One result of direct linking}

Suppose that $\lambda$ and $\mu$ are partitions of $n$ of $d$-weight $w \neq 0$, and with the same $d$-core $\gamma$. Suppose furthermore that $\mu$ is simple and disjoint from $\lambda$. Then, by definition(s), we have:

- $\mathcal{L}_{\mu}^{(i)}=\emptyset$ for $1<i \leq w$.

- $\mathcal{L}_{\lambda}^{i} \cap \mathcal{L}_{\mu}^{i}=\emptyset$ for $0 \leq i<w$.

- $\mathcal{L}_{\lambda}^{w}=\mathcal{L}_{\mu}^{w}=\{\gamma\}$.

These properties induce a large number of simplifications in the expression for the inner product of $\chi_{\lambda}$ and $\chi_{\mu}$ on $d$-singular elements. We will prove the following: 
Theorem 4.6. Suppose $\lambda$ and $\mu$ are partitions of $n$ of $d$-weight $w \neq 0$, and with the same $d$-core $\gamma$. Suppose furthermore that $\mu$ is simple and disjoint from $\lambda$. Then the unipotent characters $\chi_{\lambda}$ and $\chi_{\mu}$ are directly linked across the set of $d$-regular elements of $G$.

We write $F=\left|\mathcal{F}_{d}\right|$, where $\mathcal{F}_{d}$ is the set of irreducible monic polynomials of degree $d$ over $\mathbf{F}_{q}$, and we suppose that $F \geq n / d$ (so that $F \geq w$ for any possible $d$-weight $w$ ).

We use induction on $w$ to prove that, if $\lambda$ and $\mu$ are as above, then the inner product of $\chi^{\lambda}$ and $\chi^{\mu}$ on the set of $d$-regular elements of $G$ is

$$
<\chi^{\lambda}, \chi^{\mu}>_{d-r e g}=\frac{(-1)^{w} F^{w}}{w !\left(q^{d}-1\right)^{w}} \sum_{P_{\lambda} \in \mathcal{P}_{\gamma}^{\lambda}, P_{\mu} \in \mathcal{P}_{\gamma}^{\mu}}(-1)^{L_{P_{\lambda}}}(-1)^{L_{P_{\mu}}},
$$

where $\mathcal{P}_{\gamma}^{\lambda}$ (resp. $\mathcal{P}_{\gamma}^{\mu}$ ) denotes the set of paths from $\lambda$ (resp. $\mu$ ) to $\gamma$, in the lattice of partitions, obtained by successive removals of $d$-hooks, and, for $P_{\lambda} \in \mathcal{P}_{\gamma}^{\lambda}$ (resp. $\left.P_{\mu} \in \mathcal{P}_{\gamma}^{\mu}\right), L_{P_{\lambda}}$ (resp. $L_{P_{\mu}}$ ) is the sum of the leg lengths of the hooks removed along the corresponding path.

We suppose the result established for partitions of $d$-weight up to $w-1 \neq 0$, and we take $\lambda$ and $\mu$ of weight $w$ as above. We order the elements of $\mathcal{F}_{d}$, and write $\mathcal{F}_{d}=\left\{f_{1}, \ldots, f_{F}\right\}$. We write $\mathcal{C}$ for the set of $d$-singular elements of weight at most $w$ of $G$. The conjugacy classes in $\mathcal{C}$ are parametrized by the $F$-tuples of (possibly empty) partitions $\left(\nu_{1}, \ldots, \nu_{F}\right)$, where $\nu_{i} \vdash k_{i}$ for $1 \leq i \leq F$ and $1 \leq k_{1}+\cdots+k_{F} \leq w$. We choose a basis for $V$ and identify $G=G L(V)$ with $G L(n, q)$. We will write $G_{n}$ for $G L(n, q)$, and similarly for smaller dimensions. Writing $\sim$ for conjugation, we get

$$
\mathcal{C} / \sim=\bigcup_{k=1}^{w}\left\{\left(\begin{array}{cc}
\rho & \\
& \sigma
\end{array}\right), \rho \in \mathcal{C}_{k} / \sim, \sigma \in G_{n-k d}, \sigma d-r e g\right\},
$$

where

$$
\mathcal{C}_{k} / \sim=\bigcup_{k_{1}+\cdots+k_{F}=k} \bigcup_{\nu_{1} \vdash k_{1}, \ldots, \nu_{F} \vdash k_{F}}\left\{\left(\begin{array}{ccc}
U_{\nu_{1}}\left(f_{1}\right) & \\
& \ddots & \\
& & U_{\nu_{F}}\left(f_{F}\right)
\end{array}\right)\right\} .
$$

We have

$$
<\chi^{\lambda}, \chi^{\mu}>_{d-\operatorname{sing}}=<\chi^{\lambda}, \chi^{\mu}>_{\mathcal{C}}=\sum_{k=1}^{w}<\chi^{\lambda}, \chi^{\mu}>_{\mathcal{C}_{k}}
$$

and, for $1 \leq k \leq w,<\chi^{\lambda}, \chi^{\mu}>_{\mathcal{C}_{k}}$ is equal to

$$
\sum_{k_{1}+\cdots+k_{F}=k} \sum_{\nu_{1} \vdash k_{1}, \ldots, \nu_{F} \vdash k_{F}} \sum_{\substack{\sigma \in G_{n-k d} \\ \sigma d-r e g}} \frac{\chi^{\lambda}\left(\rho^{\nu_{1}} \ldots \rho^{\nu_{F}} \sigma\right) \overline{\chi^{\mu}\left(\rho^{\nu_{1}} \ldots \rho^{\nu_{F}} \sigma\right)}}{\left|C_{n}\left(\rho^{\nu_{1}} \ldots \rho^{\nu_{F}} \sigma\right)\right|},
$$

where we write $C_{n}(g)$ for $C_{G_{n}}(g)=C_{G L(n, q)}(g)$ and $\rho^{\nu}$ for $U_{\nu}(f), f \in \mathcal{F}$ (abuse justified since the values of unipotent characters on $U_{\nu}(f)$ and the size of its centralizer don't depend on $f$, but only on $\nu$ ). 
Repeated use of the Murnaghan-Nakayama Rule gives us

$$
\chi^{\lambda}\left(\rho^{\nu_{1}} \ldots \rho^{\nu_{F}} \sigma\right)=\sum_{\lambda_{1} \in \mathcal{L}_{\lambda}^{k_{1}}, \lambda_{2} \in \mathcal{L}_{\lambda_{1}}^{k_{2}}, \ldots, \lambda_{F} \in \mathcal{L}_{\lambda_{F-1}}^{k_{F}}} a_{\lambda_{\lambda_{1}}}^{\nu_{1}} a_{\lambda_{1} \lambda_{2}}^{\nu_{2}} \ldots a_{\lambda_{F}-1 \lambda_{F}}^{\nu_{F}} \chi^{\lambda_{F}}(\sigma),
$$

and a similar expression for $\chi^{\mu}\left(\rho^{\nu_{1}} \ldots \rho^{\nu_{F}} \sigma\right)$.

We obtain

$$
<\chi^{\lambda}, \chi^{\mu}>_{d-s i n g}=\sum_{k=1}^{w} \sum_{k_{1}+\cdots+k_{F}=k} \mathcal{A}_{k_{1}, \ldots, k_{F}}^{\lambda, \mu},
$$

where

$$
\mathcal{A}_{k_{1}, \ldots, k_{F}}^{\lambda, \mu}=\sum_{\lambda_{1} \in \mathcal{L}_{\lambda}^{k_{1}}, \ldots, \lambda_{F} \in \mathcal{L}_{\lambda_{F-1}}^{k_{F}}} \sum_{\mu_{1} \in \mathcal{L}_{\mu}^{k_{1}}, \ldots, \mu_{F} \in \mathcal{L}_{\mu_{F}-1}^{k_{F}}} \mathcal{B}_{\lambda_{1}, \mu_{1}}^{\lambda, \mu} \ldots \mathcal{B}_{\lambda_{F}, \mu_{F}}^{\lambda_{F-1}, \mu_{F-1}} \mathcal{D}_{\lambda_{F}, \mu_{F}},
$$

with

$$
\mathcal{B}_{\lambda_{1}, \mu_{1}}^{\lambda, \mu}=\sum_{\nu_{1} \vdash k_{1}} \frac{a_{\lambda \lambda_{1}}^{\nu_{1}} a_{\mu \mu_{1}}^{\nu_{1}}}{\left|C_{k_{1} d}\left(\rho^{\nu_{1}}\right)\right|}, \ldots, \mathcal{B}_{\lambda_{F}, \mu_{F}}^{\lambda_{F-1}, \mu_{F-1}}=\sum_{\nu_{F} \vdash k_{F}} \frac{a_{\lambda_{F-1} \lambda_{F}}^{\nu_{F}} a_{\mu_{F-1} \mu_{F}}^{\nu_{F}}}{\left|C_{k_{F} d}\left(\rho^{\nu_{F}}\right)\right|}
$$

and

$$
\mathcal{D}_{\lambda_{F}, \mu_{F}}=\sum_{\substack{\sigma \in G_{n-k d} / \sim \\ \sigma d-r e g}} \frac{\chi^{\lambda_{F}}(\sigma) \overline{\chi^{\mu_{F}}(\sigma)}}{\left|C_{n-k d}(\sigma)\right|} .
$$

Now, for all $1 \leq k \leq w$, we have

$$
\sum_{\substack{\sigma \in G_{n-k d} / \sim \\ \sigma d-r e g}} \frac{\chi^{\lambda_{F}}(\sigma) \overline{\chi^{\mu_{F}}(\sigma)}}{\left|C_{n-k d}(\sigma)\right|}=<\chi^{\lambda_{F}}, \chi^{\mu_{F}}>_{G_{n-k d}, d-r e g},
$$

which is known, by the induction hypothesis, since $\lambda_{F}$ and $\mu_{F}$ have weight $w-k<w$ (and the same $d$-core $\gamma$ ).

In order to deal with the other factors, we start with the following lemma, which doesn't depend on the special choice of $\mu$ :

Lemma 4.7. For any $1 \leq i \leq F$, we have, writing $\lambda_{0}$ and $\mu_{0}$ for $\lambda$ and $\mu$ respectively,

$$
\sum_{\nu_{i} \vdash k_{i}} \frac{a_{\lambda_{i-1} \lambda_{i}}^{\nu_{i}} \mu_{\mu_{i-1} \mu_{i}}^{\nu_{i}}}{\left|C_{k_{i}} d\left(\rho^{\nu_{i}}\right)\right|}=\varepsilon_{\lambda_{i-1} \lambda_{i}} \varepsilon_{\mu_{i-1} \mu_{i}} \sum_{\alpha \vdash k_{i}} \frac{\phi_{\lambda_{i-1} \mid \lambda_{i}}(\alpha) \phi_{\mu_{i-1} \mid \mu_{i}}(\alpha)}{\left|C_{S_{k_{i}}}(\alpha)\right|\left|T_{\bar{\alpha}}\right|}
$$

where:

- if $\alpha=\left(1^{r_{1}}, 2^{r_{2}}, \ldots, s^{r_{s}}\right) \vdash k_{i}$, then $\bar{\alpha}=\left(d^{r_{1}},(2 d)^{r_{2}}, \ldots,(s d)^{r_{s}}\right) \vdash k_{i} d$,

- $T_{\bar{\alpha}}$ is a maximal torus of $G_{k_{i} d}=G L\left(k_{i} d, q\right)$ representing the $G_{k_{i} d^{-}}$-conjugacy class corresponding to the $S_{k_{i}}$-conjugacy class of $\alpha$; we have $\left|T_{\bar{\alpha}}\right|=\prod_{i}\left(q^{d i}-1\right)^{r_{i}}$ (cf [5] (1.12)); 
- $\phi_{\mu_{i-1} \mid \mu_{i}} \in \mathbf{Z} \operatorname{Irr}\left(S_{k_{i}}\right)$, and, if $h \in S_{\ell}$ and $g \in S_{k_{i} d}$ is of cycle type $\bar{\alpha}$ $\left(\alpha \vdash k_{i}\right)$, then

$$
\varphi_{\mu_{i-1}}(g h)=\sum_{\eta \in \mathcal{L}_{\mu_{i-1}}^{k_{i}}} \varepsilon_{\mu_{i-1} \eta} \phi_{\mu_{i-1} \mid \eta}(\alpha) \varphi_{\eta}(h)
$$

(where, for any positive integer $m$, we write $\operatorname{Irr}\left(S_{m}\right)=\left\{\varphi_{\zeta}, \zeta \vdash m\right\}$ ) and similarly for $\phi_{\lambda_{i-1} \mid \lambda_{i}}$ (cf [4] and [5] (2.3)).

Proof. For all $\nu_{i} \vdash k_{i}$, we have $C_{k_{i} d}\left(\rho^{\nu_{i}}\right)=C_{C_{k_{i} d}\left(\rho_{S}^{\nu_{i}}\right)}\left(\rho_{U}^{\nu_{i}}\right)$, where $\rho_{S}^{\nu_{i}}$ and $\rho_{U}^{\nu_{i}}$ are the semisimple and unipotent parts of $\rho^{\nu_{i}}$ respectively, and $C_{k_{i} d}\left(\rho_{S}^{\nu_{i}}\right) \cong$ $G L\left(k_{i}, q^{d}\right)$. We write $H_{i}$ for $G L\left(k_{i}, q^{d}\right)$ (then $H_{i}$ has Weyl group $W_{H_{i}} \cong S_{k_{i}}$ ), so that $C_{k_{i} d}\left(\rho^{\nu_{i}}\right) \cong C_{H_{i}}\left(\rho_{U}^{\nu_{i}}\right)$.

We have, writing $H_{i}^{u} / \sim$ for a set of representatives for the unipotent conjugacy classes of $H_{i}$,

$$
\sum_{\nu_{i} \vdash k_{i}} \frac{a_{\lambda_{i-1} \lambda_{i}}^{\nu_{i}} a_{\mu_{i-1} \mu_{i}}^{\nu_{i}}}{\left|C_{k_{i} d}\left(\rho^{\nu_{i}}\right)\right|}=\sum_{\rho_{U}^{\nu_{i}} \in H_{i}^{u} / \sim} \frac{a_{\lambda_{i-1} \lambda_{i}}^{\nu_{i}} a_{\mu_{i-1} \mu_{i}}^{\nu_{i}}}{\left|C_{H_{i}}\left(\rho_{U}^{\nu_{i}}\right)\right|} .
$$

Now, by definition ( $\mathrm{cf}[5$ Theorem $(2 \mathrm{G})$ ), we have

$$
a_{\lambda_{i-1} \lambda_{i}}^{\nu_{i}}=\varepsilon_{\lambda_{i-1} \lambda_{i}} \sum_{\alpha \vdash k_{i}} \frac{1}{\left|W_{H_{i}}\left(T_{\bar{\alpha}}\right)\right|} Q_{T_{\bar{\alpha}}}^{H_{i}}\left(\rho_{U}^{\nu_{i}}\right) \phi_{\lambda_{i-1} \mid \lambda_{i}}(\alpha)
$$

(and similarly for $a_{\mu_{i-1} \mu_{i}}^{\nu_{i}}$ ), where $W_{H_{i}}\left(T_{\bar{\alpha}}\right)$ is the Weyl group of $T_{\bar{\alpha}}$ in $H_{i}$, so that $\left|W_{H_{i}}\left(T_{\bar{\alpha}}\right)\right|=\left|C_{S_{k_{i}}}(\alpha)\right|$ (cf [5] (1.11)), and $Q_{T_{\bar{\alpha}}}^{H_{i}}$ is the Green function, integer-valued class function defined on $H_{i}^{u}$, associated to $T_{\bar{\alpha}}$ (cf [5]).

We obtain that $\sum_{\nu_{i} \vdash k_{i}} \frac{a_{\lambda_{i-1} \lambda_{i}}^{\nu_{i}} a_{\mu_{i-1} \mu_{i}}^{\nu_{i}}}{\left|C_{k_{i}} d\left(\rho^{\nu_{i}}\right)\right|}$ is equal to

$$
\sum_{\rho_{U}^{\nu_{i}} \in H_{i}^{u} / \sim} \frac{\varepsilon_{\lambda_{i-1} \lambda_{i}} \varepsilon_{\mu_{i-1} \mu_{i}}}{\left|C_{H_{i}}\left(\rho_{U}^{\nu_{i}}\right)\right|} \sum_{\alpha, \beta \vdash k_{i}} \frac{Q_{T_{\bar{\alpha}}}^{H_{i}}\left(\rho_{U}^{\nu_{i}}\right) \phi_{\lambda_{i-1} \mid \lambda_{i}}(\alpha) Q_{T_{\bar{\beta}}}^{H_{i}}\left(\rho_{U}^{\nu_{i}}\right) \phi_{\mu_{i-1} \mid \mu_{i}}(\beta)}{\left|W_{H_{i}}\left(T_{\bar{\alpha}}\right)\right|\left|W_{H_{i}}\left(T_{\bar{\beta}}\right)\right|},
$$

which, since the Green functions are class functions, can be written as

$$
\varepsilon_{\lambda_{i-1} \lambda_{i}} \varepsilon_{\mu_{i-1} \mu_{i}} \sum_{\alpha, \beta \vdash k_{i}} \frac{\phi_{\lambda_{i-1} \mid \lambda_{i}}(\alpha) \phi_{\mu_{i-1} \mid \mu_{i}}(\beta)}{\left|W_{H_{i}}\left(T_{\bar{\alpha}}\right)\right|\left|W_{H_{i}}\left(T_{\bar{\beta}}\right)\right|}\left(\frac{1}{\left|H_{i}\right|} \sum_{\rho_{U}^{\nu_{i} \in H_{i}^{u}}} Q_{T_{\bar{\alpha}}}^{H_{i}}\left(\rho_{U}^{\nu_{i}}\right) Q_{T_{\bar{\beta}}}^{H_{i}}\left(\rho_{U}^{\nu_{i}}\right)\right) .
$$

Now, by the first orthogonality relation for Green functions in $H_{i}(\mathrm{cf}[5]$ (1.7)), we have

$$
\frac{1}{\left|H_{i}\right|} \sum_{\rho_{U}^{\nu_{i} \in H_{i}^{u}}} Q_{T_{\bar{\alpha}}}^{H_{i}}\left(\rho_{U}^{\nu_{i}}\right) Q_{T_{\bar{\beta}}}^{H_{i}}\left(\rho_{U}^{\nu_{i}}\right)= \begin{cases}\frac{\left|W_{H_{i}}\left(T_{\bar{\alpha}}\right)\right|}{\left|T_{\bar{\alpha}}\right|} & \text { if } T_{\bar{\alpha}} \sim_{H_{i}} T_{\bar{\beta}} \\ 0 & \text { otherwise }\end{cases}
$$

But $T_{\bar{\alpha}} \sim_{H_{i}} T_{\bar{\beta}}$ if and only if $\bar{\alpha}=\bar{\beta}$, if and only if $\alpha=\beta$. We thus get, since $\left|W_{H_{i}}\left(T_{\bar{\alpha}}\right)\right|=\left|C_{S_{k_{i}}}(\alpha)\right|$,

$$
\sum_{\nu_{i} \vdash k_{i}} \frac{a_{\lambda_{i-1} \lambda_{i}}^{\nu_{i}} a_{\mu_{i-1} \mu_{i}}^{\nu_{i}}}{\left|C_{k_{i} d}\left(\rho^{\nu_{i}}\right)\right|}=\varepsilon_{\lambda_{i-1} \lambda_{i}} \varepsilon_{\mu_{i-1} \mu_{i}} \sum_{\alpha \vdash k_{i}} \frac{\phi_{\lambda_{i-1} \mid \lambda_{i}}(\alpha) \phi_{\mu_{i-1} \mid \mu_{i}}(\alpha)}{\left|C_{S_{k_{i}}}(\alpha)\right|\left|T_{\bar{\alpha}}\right|},
$$

which ends the proof. 
The fact that $\mu$ is simple then gives us further simplifications; we have the following

Lemma 4.8. For any $1 \leq i \leq F$ and $\alpha \in S_{k_{i}}$, we have $\phi_{\mu_{i-1} \mid \mu_{i}}(\alpha)=0$ unless $\alpha=\left(1^{k_{i}}\right)$ (the identity element of $S_{k_{i}}$ ).

Proof. Take any $1 \leq i \leq F$. We have $\mu_{i-1} \vdash m$ and $\mu_{i} \vdash \ell$, with $m=\ell+k_{i} d$. Then, by definition (cf [5] (2.3) and [4]), $\phi_{\mu_{i-1} \mid \mu_{i}} \in \operatorname{ZIrr}\left(S_{k_{i}}\right)$, and, if $h \in S_{\ell}$ and $g \in S_{k_{i} d}$ is of cycle type $\bar{\alpha}\left(\alpha \vdash k_{i}\right)$, then

$$
\varphi_{\mu_{i-1}}(g h)=\sum_{\eta \in \mathcal{L}_{\mu_{i-1}}^{k_{i}}} \varepsilon_{\mu_{i-1} \eta} \phi_{\mu_{i-1} \mid \eta}(\alpha) \varphi_{\eta}(h) .
$$

Now $\mu_{i-1}$ can be obtained from $\mu$, which is $d$-simple, by removing a sequence of $d$-hooks, so that $\mu_{i-1}$ is itself simple, whence, for $j>1$, there is no $j d$-hook in $\mu_{i-1}$.

Thus, by the Murnaghan-Nakayama rule (in the symmetric group), if there is a cycle of length $j>1$ in $\alpha$ (so that there is a $j d$-cycle in $g$ ), then

$$
\varphi_{\mu_{i-1}}(g h)=0 \text {, for all } h \in S_{\ell} .
$$

Hence, for all $h \in S_{\ell}$,

$$
\sum_{\eta \in \mathcal{L}_{\mu_{i-1}}^{k_{i}}} \varepsilon_{\mu_{i-1} \eta} \phi_{\mu_{i-1} \mid \eta}(\alpha) \varphi_{\eta}(h)=0
$$

whence

$$
\varepsilon_{\mu_{i-1} \eta} \phi_{\mu_{i-1} \mid \eta}(\alpha)=0 \text { for all } \eta \in \mathcal{L}_{\mu_{i-1}}^{k_{i}},
$$

in particular for $\eta=\mu_{i}$.

By the previous two lemmas, we therefore get, for any $1 \leq i \leq F$,

$$
\mathcal{B}_{\lambda_{i}, \mu_{i}}^{\lambda_{i-1}, \mu_{i-1}}=\frac{\varepsilon_{\lambda_{i-1} \lambda_{i}} \varepsilon_{\mu_{i-1} \mu_{i}} \phi_{\lambda_{i-1} \mid \lambda_{i}}\left(1^{k_{i}}\right) \phi_{\mu_{i-1} \mid \mu_{i}}\left(1^{k_{i}}\right)}{\left|C_{S_{k_{i}}}\left(1^{k_{i}}\right)\right|\left|T \overline{\left(1^{k_{i}}\right)}\right|},
$$

and we have $C_{S_{k_{i}}}\left(1^{k_{i}}\right)=S_{k_{i}}$ of order $k_{i}$ ! , and $\left|T_{\overline{1^{k_{i}}}}\right|=\left|T_{\left(d^{k_{i}}\right)}\right|=\left(q^{d}-1\right)^{k_{i}}$.

Now, we have, for all $h \in S_{\ell}$,

$$
\varphi_{\mu_{i-1}}\left(\overline{1^{k_{i}}} h\right)=\sum_{\eta \in \mathcal{L}_{\mu_{i-1}}^{k_{i}}} \varepsilon_{\mu_{i-1} \eta} \phi_{\mu_{i-1} \mid \eta}\left(1^{k_{i}}\right) \varphi_{\eta}(h) .
$$

On the other hand, by repeated use of the Murnaghan-Nakayama Rule, we have, for all $h \in S_{\ell}$,

$$
\varphi_{\mu_{i-1}}\left(\overline{1^{k_{i}}} h\right)=\sum_{\eta \in \mathcal{L}_{\mu_{i-1}}^{k_{i}}}\left(\sum_{P \in \mathcal{P}_{\eta}^{\mu_{i-1}}}(-1)^{L_{P}}\right) \varphi_{\eta}(h) .
$$

Hence, for all $\eta \in \mathcal{L}_{\mu_{i-1}}^{k_{i}}$,

$$
\varepsilon_{\mu_{i-1} \eta} \phi_{\mu_{i-1} \mid \eta}\left(1^{k_{i}}\right)=\sum_{P \in \mathcal{P}_{\eta}^{\mu_{i-1}}}(-1)^{L_{P}} .
$$


Similarly, for all $\eta \in \mathcal{L}_{\lambda_{i-1}}^{k_{i}}$,

$$
\varepsilon_{\lambda_{i-1} \eta} \phi_{\lambda_{i-1} \mid \eta}\left(1^{k_{i}}\right)=\sum_{P \in \mathcal{P}_{\eta}^{\lambda_{i-1}}}(-1)^{L_{P}} .
$$

We therefore have, for any $1 \leq i \leq F$,

$$
\mathcal{B}_{\lambda_{i}, \mu_{i}}^{\lambda_{i-1}, \mu_{i-1}}=\sum_{P_{\lambda_{i}} \in \mathcal{P}_{\lambda_{i}}^{\lambda_{i-1}}, P_{\mu_{i}} \in \mathcal{P}_{\mu_{i}}^{\mu_{i-1}}} \frac{(-1)^{L_{P_{\lambda_{i}}}(-1)^{L_{P_{\mu_{i}}}}}}{k_{i} !\left(q^{d}-1\right)^{k_{i}}}
$$

Coming back to our expression for $\left\langle\chi^{\lambda}, \chi^{\mu}>_{d-s i n g}\right.$, we see that, for each $1 \leq k \leq w$, once $k_{1}, \ldots, k_{F}$ are chosen, then all the paths obtained by removing a sequence of $k d$-hooks from $\lambda$ and $\mu$ appear exactly once in the sum. We get that $\mathcal{A}_{k_{1}, \ldots, k_{F}}^{\lambda, \mu}$ is equal to

$$
\frac{1}{k_{1} ! \ldots k_{F} !\left(q^{d}-1\right)^{k}} \sum_{\substack{\lambda^{\prime} \in \mathcal{L}_{\lambda}^{k} \\ \mu^{\prime} \in \mathcal{L}_{\mu}^{k}}} \sum_{\substack{P_{\lambda} \in \mathcal{P}_{\lambda^{\prime}}^{\lambda} \\ P_{\mu} \in \mathcal{P}_{\mu^{\prime}}^{\mu^{\prime}}}}(-1)^{L_{P_{\lambda}}}(-1)^{L_{P_{\mu}}}<\chi^{\lambda^{\prime}}, \chi^{\mu^{\prime}}>_{d-r e g} .
$$

Now, for all $\lambda^{\prime} \in \mathcal{L}_{\lambda}^{k}$ and $\mu^{\prime} \in \mathcal{L}_{\mu}^{k}, \lambda^{\prime}$ and $\mu^{\prime}$ have $d$-weight $w-k$ and $d$-core $\gamma$, and $\mu^{\prime}$ is simple and disjoint from $\lambda^{\prime}$. By the induction hypothesis, we thus have

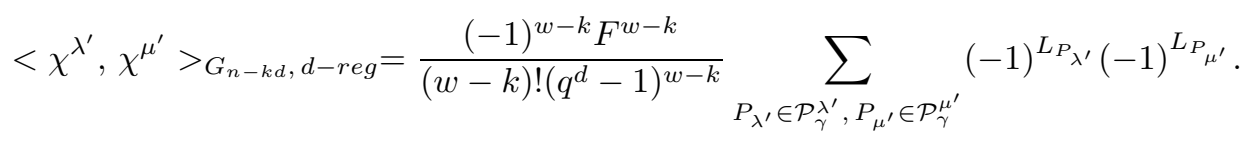

Finally, we get

$$
<\chi^{\lambda}, \chi^{\mu}>_{d-s i n g}=\sum_{k=1}^{w} \sum_{k_{1}+\cdots+k_{F}=k} \mathcal{A}_{k_{1}, \ldots, k_{F}}^{\lambda, \mu},
$$

where

$$
\mathcal{A}_{k_{1}, \ldots, k_{F}}^{\lambda, \mu}=\frac{(-1)^{w-k} F^{w-k}}{k_{1} ! \ldots k_{F} !(w-k) !\left(q^{d}-1\right)^{w}} \sum_{P_{\lambda} \in \mathcal{P}_{\gamma}^{\lambda}, P_{\mu} \in \mathcal{P}_{\gamma}^{\mu}}(-1)^{L_{P_{\lambda}}}(-1)^{L_{P_{\mu}}} .
$$

In order to prove our result, it therefore suffices to show that

$$
\sum_{k=1}^{w} \sum_{k_{1}+\cdots+k_{F}=k} \frac{(-1)^{w-k} F^{w-k}}{k_{1} ! \ldots k_{F} !(w-k) !}=\frac{(-1)^{w+1} F^{w}}{w !} .
$$

We rewrite the second sum in the left hand side as a sum over the partitions of $k$. When doing this, we "break" the ordering on the elements of $\mathcal{F}_{d}$, so that we have to count how many times each given partition appears once its parts are fixed, and divide by the number of times the corresponding conjugacy class of $d$-elements appears. More precisely, to each partition $\left(k^{r_{k}}, \ldots, 1^{r_{1}}\right)$ of $k$ with $r_{1}+\cdots+r_{k}=r$ parts, there are $F(F-1) \cdots(F-r+1)$ ways to associate one distinct polynomial of $\mathcal{F}_{d}$ to each part (which is why we supposed that $F \geq w$ ), but (since the order of the polynomials doesn't matter, only their multiplicity 
(i.e. $\left(\begin{array}{ll}f & \\ & g\end{array}\right)$ and $\left(\begin{array}{ll}g & \\ & f\end{array}\right)$ are conjugate)) each conjugacy class of $d$-element of type $\left(k^{r_{k}}, \ldots, 1^{r_{1}}\right)$ will appear $r_{1} ! \ldots r_{k}$ ! times in this way. The left hand side thus becomes

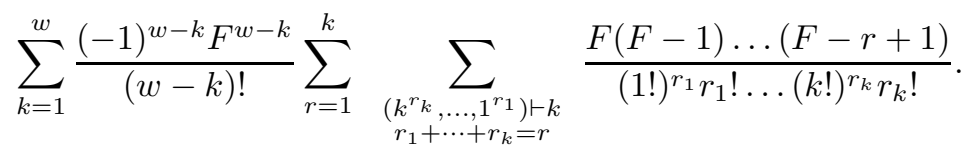

To conclude, we just need the following:

Lemma 4.9. For any integers $k \geq 1$ and $F \geq k$, we have

$$
\sum_{r=1}^{k} \sum_{\substack{\left.k^{r_{k}}, \ldots, 1^{r_{1}}\right) \vdash k \\ r_{1}+\cdots+r_{k}=r}} \frac{F(F-1) \ldots(F-r+1)}{(1 !)^{r_{1}} r_{1} ! \ldots(k !)^{r_{k}} r_{k} !}=\frac{F^{k}}{k !} .
$$

Proof. The coefficient of $z^{k}$ in the power series of $\exp (z)^{F}$ is $F^{k} / k !$. Indeed,

$$
\exp (z)^{F}=\exp (F z)=\sum_{n \geq 0} \frac{F^{n}}{n !} z^{n} .
$$

On the other hand, we have

$$
\left(\sum_{n \geq 0} \frac{1}{n !} z^{n}\right)^{F}=\sum_{r_{0}+r_{1}+\cdots=F} \frac{C_{\left(r_{0}, r_{1}, \ldots\right)}}{(0 !)^{r_{0}}(1 !)^{r_{1}} \ldots} z^{\sum_{i \geq 0} i r_{i}},
$$

where $C_{\left(r_{0}, r_{1}, \ldots\right)}$ is the multinomial coefficient $\frac{F !}{r_{0} ! r_{1} ! \ldots}$. The coefficient of $z^{k}$ in this expression therefore corresponds to all the uplets $\left(r_{0}, r_{1}, \ldots\right)$ such that $r_{0}+r_{1}+\cdots=F$ and $\sum_{i \geq 0} i r_{i}=\sum_{i>0} i r_{i}=k$. Hence $\left(1^{r_{1}}, \ldots\right) \vdash k$ is a partition with $\sum_{i>0} r_{i}=: r$ parts, $r_{0}=F-\sum_{i>0} r_{i}=F-r$, and $C_{\left(r_{0}, r_{1}, \ldots\right)}=\frac{F !}{(F-r) ! r_{1} ! \ldots}$. Finally, we get that the coefficient of $z^{k}$ is

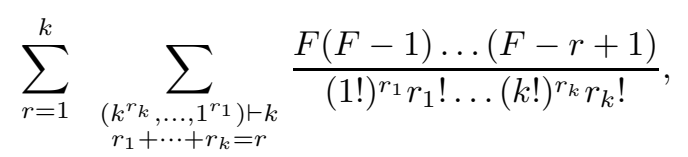

which is the desired result.

Remark: In the proof, we have used the fact that $F$ is an integer. However, the equality given for each integer $k \geq 1$ in the statement of the lemma is an equality between two polynomial functions in $F$, satisfied for an infinite number of values of $F$. It is therefore in fact an equality in the polynomial ring $\mathbf{Q}[F]$. 
Using the previous lemma, we get

$$
\begin{aligned}
\sum_{k=1}^{w} \sum_{k_{1}+\cdots+k_{F}=k} \frac{(-1)^{w-k} F^{w-k}}{k_{1} ! \ldots k_{F} !(w-k) !} & =\sum_{k=1}^{w} \frac{(-1)^{w-k} F^{w-k}}{(w-k) !} \frac{F^{k}}{k !} \\
& =\frac{F^{w}}{w !} \sum_{k=1}^{w} \frac{(-1)^{w-k} w !}{k !(w-k) !} \\
& =\frac{(-1)^{w} F^{w}}{w !} \sum_{k=1}^{w}(-1)^{k}\left(\begin{array}{c}
w \\
k
\end{array}\right) \\
& =\frac{(-1)^{w} F^{w}}{w !}\left[(1-1)^{w}-1\right] \\
& =\frac{(-1)^{w+1} F^{w}}{w !} .
\end{aligned}
$$

Putting everything back together, we finally obtain

$$
<\chi^{\lambda}, \chi^{\mu}>_{d-s i n g}=\frac{(-1)^{w+1} F^{w}}{w !\left(q^{d}-1\right)^{w}} \sum_{P_{\lambda} \in \mathcal{P}_{\gamma}^{\lambda}, P_{\mu} \in \mathcal{P}_{\gamma}^{\mu}}(-1)^{L_{P_{\lambda}}}(-1)^{L_{P_{\mu}}},
$$

which is equivalent to

$$
<\chi^{\lambda}, \chi^{\mu}>_{d-r e g}=\frac{(-1)^{w} F^{w}}{w !\left(q^{d}-1\right)^{w}} \sum_{P_{\lambda} \in \mathcal{P}_{\gamma}^{\lambda}, P_{\mu} \in \mathcal{P}_{\gamma}^{\mu}}(-1)^{L_{P_{\lambda}}(-1)^{L_{P_{\mu}}}} .
$$

To conclude by induction, it only remains to study the case $w=1$. This case can in fact be treated without the simplicity hypothesis on $\mu$.

We take $\lambda$ and $\mu$, distinct partitions of $n$ of $d$-weight 1 , and with the same $d$-core $\gamma$. Then $\mathcal{L}_{\lambda}^{1}=\mathcal{L}_{\mu}^{1}=\{\gamma\}$, and $\mathcal{L}_{\lambda}^{i}=\mathcal{L}_{\mu}^{i}=\emptyset$ for $i>1$, and we write $\mathcal{P}_{\gamma}^{\lambda}=\left\{P_{\lambda}\right\}$ and $\mathcal{P}_{\gamma}^{\mu}=\left\{P_{\mu}\right\}$. As above, it suffices to consider the inner product of $\chi^{\lambda}$ and $\chi^{\mu}$ on the set of $d$-singular elements of $d$-weight at most 1 . The minimal polynomial of any such element has exactly one irreducible factor of degree $d$, and it has multiplicity one. Any $d$-singular element $g \in G$ of weight 1 can thus be written uniquely $g=\rho \sigma=\sigma \rho$, where $\rho$ can be seen as a $d$-element of $G_{d}=G L(d, q)$, and $\sigma$ as a $d$-regular element of $G_{n-d}=G L(n-d, q)$.

We have

$$
\begin{aligned}
<\chi^{\lambda}, \chi^{\mu}>_{d-s i n g} & =\sum_{\substack{\rho \in G_{d} / \sim \\
\rho d-\operatorname{sing}}} \sum_{\substack{\sigma \in G_{n-d} / \sim \\
\sigma d-r e g}} \frac{1}{\left|C_{G_{n}}(\rho \sigma)\right|} \chi^{\lambda}(\rho \sigma) \chi^{\mu}(\rho \sigma) \\
& =\sum_{\substack{\rho \in G_{d} / \sim \\
\rho d-\operatorname{sing}}} \frac{a_{\lambda \gamma}^{\rho} a_{\mu \gamma}^{\rho}}{\left|C_{G_{d}}(\rho)\right|} \sum_{\substack{\sigma \in G_{n-d} / \sim \\
\sigma d-r e g}} \frac{\chi^{\lambda}(\rho \sigma) \chi^{\mu}(\rho \sigma)}{\left|C_{G_{n-d}}(\sigma)\right|} \\
& =F \sum_{\nu \vdash 1} \frac{a_{\lambda \gamma}^{\rho^{\nu}} a_{\mu \gamma}^{\rho^{\nu}}}{\left|C_{G_{d}}\left(\rho^{\nu}\right)\right|}<\chi^{\gamma}, \chi^{\gamma}>_{G_{n-d}, d-r e g} .
\end{aligned}
$$

By Lemma 4.7, we thus have

$$
\begin{aligned}
<\chi^{\lambda}, \chi^{\mu}>_{d-s i n g} & =F \frac{\varepsilon_{\lambda \gamma} \varepsilon_{\mu \gamma} \phi_{\lambda \mid \gamma}(1) \phi_{\mu \mid \gamma}(1)}{\left|C_{S_{1}}(1)\right||T \overline{(1)}|}<\chi^{\gamma}, \chi^{\gamma}>_{G_{n-d}, d-r e g} \\
& =F \frac{\varepsilon_{\lambda \gamma} \varepsilon_{\mu \gamma} \phi_{\lambda \mid \gamma}(1) \phi_{\mu \mid \gamma}(1)}{q^{d}-1}<\chi^{\gamma}, \chi^{\gamma}>_{G_{n-d}, d-r e g} \\
& =F \frac{\varepsilon_{\lambda \gamma} \varepsilon_{\mu \gamma} \phi_{\lambda \mid \gamma}(1) \phi_{\mu \mid \gamma}(1)}{q^{d}-1}
\end{aligned}
$$


since $\gamma$ is a $d$-core, whence vanishes on $d$-singular elements of $G L(n-d, q)$. Finally, as after Lemma 4.8 , we have $\varepsilon_{\lambda \gamma} \phi_{\lambda \mid \gamma}(1)=(-1)^{L_{P_{\lambda}}}$ and $\varepsilon_{\mu \gamma} \phi_{\mu \mid \gamma}(1)=$ $(-1)^{L_{P_{\mu}}}$, which gives us

$$
<\chi^{\lambda}, \chi^{\mu}>_{d-s i n g}=\frac{F}{q^{d}-1}(-1)^{L_{P_{\lambda}}}(-1)^{L_{P_{\mu}}} .
$$

Hence the result is true for $w=1$. Thus, by induction, we have that, for any $w \geq 1$, if $\lambda$ and $\mu$ are partitions of $n$ of $d$-weight $w$, with the same $d$-core $\gamma$, and if $\mu$ is simple and disjoint from $\lambda$, then

$$
<\chi^{\lambda}, \chi^{\mu}>_{d-r e g}=\frac{(-1)^{w} F^{w}}{w !\left(q^{d}-1\right)^{w}} \sum_{P_{\lambda} \in \mathcal{P}_{\gamma}^{\lambda}, P_{\mu} \in \mathcal{P}_{\gamma}^{\mu}}(-1)^{L_{P_{\lambda}}}(-1)^{L_{P_{\mu}}} .
$$

To conclude, we just note that, in fact, $(-1)^{L_{P_{\lambda}}}$ (resp. $(-1)^{L_{P_{\mu}}}$ ) is independent on the path $P_{\lambda} \in \mathcal{P}_{\gamma}^{\lambda}$ (resp. $P_{\mu} \in \mathcal{P}_{\gamma}^{\mu}$ ) (cf [7], Theorem 2.7.27). We therefore write $(-1)^{L_{P_{\lambda}}}=\varepsilon_{\lambda}$ for any $P_{\lambda} \in \mathcal{P}_{\gamma}^{\lambda}$ (resp. $(-1)^{L_{P_{\mu}}}=\varepsilon_{\mu}$, for any $\left.P_{\mu} \in \mathcal{P}_{\gamma}^{\mu}\right)$. We get

$$
<\chi^{\lambda}, \chi^{\mu}>_{d-r e g}=\frac{(-1)^{w} F^{w}}{w !\left(q^{d}-1\right)^{w}}\left|\mathcal{P}_{\gamma}^{\lambda}\right|\left|\mathcal{P}_{\gamma}^{\mu}\right| \varepsilon_{\lambda} \varepsilon_{\mu} \neq 0 .
$$

This ends the proof of Theorem 4.6.

\subsection{Nakayama Conjecture for blocks of small weight}

We are now in position to prove the second direction of the Nakayama Conjecture for unipotent $d$-blocks of $G$, provided their weight is "small" compared to $d$. For partitions $\lambda$ and $\mu$ of $n$, we will write $\lambda \sim \mu$ if $\chi_{\lambda}$ and $\chi_{\mu}$ are directly linked across $d$-singular elements, and $\lambda \equiv \mu$ if $\chi_{\lambda}$ and $\chi_{\mu}$ belong to the same unipotent $d$-block of $G$. First, take $\lambda$ and $\mu$ of weight $w \neq 0$, with the same $d$-core, and suppose that $3 w \leq d$ and $F \geq w$. Then there exists $\nu \vdash n$ which is simple and disjoint from both $\lambda$ and $\mu$. Thus, by Theorem 4.6, $\lambda \sim \nu$ and $\mu \sim \nu$, so that $\lambda \equiv \mu$. In fact, we can do better than that:

Theorem 4.10. Let $d>0$ be an integer. Suppose $w>2$ is an integer, and $w \leq F$ (the number of irreducible monic polynomials of degree $d$ over $\mathbf{F}_{q}$ ). If $d \geq 2 w-1$, then the unipotent $d$-blocks of $G$ of weight $w$ satisfy the Nakayama Conjecture.

Proof. Take $\lambda$ and $\mu$ partitions of $n$ of weight $w$, with the same $d$-core $\gamma$. The proof is easy to understand on a picture of the abacus, even though it is difficult to write down.

Throughout the proof, we will use the following notations: we say that $\lambda$ uses a runner of the abacus if some $d$-hook removal of $\lambda$ is stored on this runner; we say that $\mu \subset \lambda$ if all the runners used by $\mu$ are used by $\lambda$.

First, suppose that $\lambda$ and $\mu$ use each at most $w-1$ runners. Then there exists $\nu \vdash n$ which is simple and disjoint from $\lambda$, and of $d$-core $\gamma$. By Theorem 4.6, we have $\lambda \sim \nu$. Now

- if $\mu \not \subset \nu$, then there exists $\zeta \vdash n$, disjoint from $\nu$, with $d$-core $\gamma$, and which uses a single runner, which runner is also used by $\mu$. Then $\nu \sim \zeta$. Now there exists $\xi$, which is simple, disjoint from $\mu$ (and thus from $\zeta$ ), and with $d$-core $\gamma$, so that $\zeta \sim \xi$ and $\xi \sim \mu$. Hence $\lambda \equiv \mu$. 
- if $\mu \subset \nu$, then there exists $\zeta$, disjoint from $\nu$, with $d$-core $\gamma$, and which uses a single runner, which runner is not used by $\mu$. Thus $\nu \sim \zeta$. There exists $\xi$, simple, disjoint from $\zeta$, with $d$-core $\gamma$, and such that at least one runner used by $\mu$ is not used by $\xi$; we have $\zeta \sim \xi$. Then $\xi \sim \delta$, where $\delta$ has $d$-core $\gamma$, and uses a single runner, used by $\mu$ but not by $\xi$. Now $\delta \sim \eta$, simple, with $d$-core $\gamma$, and disjoint from $\mu$ (and thus from $\delta$ ). Thus $\eta \sim \mu$, and $\lambda \equiv \mu$.

Next, suppose that $\lambda$ is simple (i.e. lies on $w$ runners). If $\mu$ is disjoint from $\lambda$, then, by Theorem 4.6, $\lambda \sim \mu$, so that $\lambda \equiv \mu$. Suppose thus that $\lambda$ and $\mu$ share a runner.

- Suppose $\mu$ lies on at most $w-1$ runners.

If $\mu \subset \lambda$, then $\lambda \sim \nu$ with $d$-core $\gamma$, disjoint from $\lambda$, and using a single runner. Now $\nu \sim \zeta$, simple, with $d$-core $\gamma$, and such that $\mu \not \subset \zeta$. Then $\zeta \sim \xi$ with $d$-core $\gamma$, using a single runner, which runner is used by $\mu$. Then $\xi \sim \eta$ simple, with $d$-core $\gamma$, and disjoint from $\mu$ (and thus from $\xi$ ), so that $\eta \sim \mu$. Hence $\lambda \equiv \mu$.

If $\mu \not \subset \lambda$, then $\lambda \sim \nu$ with $d$-core $\gamma$, disjoint from $\lambda$, and using a single runner, which runner is used by $\mu$. Thus $\nu \sim \zeta$ simple, with $d$-core $\gamma$, and disjoint from $\mu$ (and thus from $\nu$ ), so that $\zeta \sim \mu$. Hence $\lambda \equiv \mu$.

- Suppose $\mu$ is simple.

If there exists a runner which is used by neither $\lambda$ nor $\mu$, then there exists $\nu$ with $d$-core $\gamma$ which lies on this single runner (and thus is disjoint from both $\lambda$ and $\mu$ ), so that $\lambda \sim \nu$ and $\nu \sim \mu$, whence $\lambda \equiv \mu$.

If not, then, necessarily, $d=2 w-1$, and $\lambda$ and $\mu$ share a single runner. Since $w>2$, there is at least one runner used by $\mu$ and not by $\lambda$. Then $\lambda \sim \nu$ with $d$-core $\gamma$ which lies on this single runner (and is thus disjoint from $\lambda$ ). Now $\nu \sim \zeta$ simple, with $d$-core $\gamma$, disjoint from $\nu$ and which doesn't use at least one runner which is used by $\lambda$ but not by $\mu$. Then $\zeta \sim \xi$ with $d$-core $\gamma$ and which lies on this single runner. In particular, $\xi$ is disjoint from $\mu$, so that $\xi \sim \mu$ (since $\mu$ is simple). Hence $\lambda \equiv \mu$.

This concludes the proof.

\section{Remarks:}

1. The case of blocks of weight 0 is easily dealt with. A partition of $d$-weight 0 is its own $d$-core, so that the corresponding unipotent character is alone in its unipotent $d$-block. Hence, for any $d>0$, the unipotent $d$-blocks of $G$ of weight 0 satisfy the Nakayama Conjecture.

2. As we have remarked earlier, in the case $w=1$, we don't need to use simple partitions. For any $d>0$ and for any two partitions $\lambda$ and $\mu$ of $n$ of $d$-weight 1 and with the same $d$-core, the unipotent characters $\chi_{\lambda}$ and $\chi_{\mu}$ are directly linked across $d$-singular elements. Hence the unipotent $d$-blocks of weight 1 of $G$ satisfy the Nakayama Conjecture.

3 . As can be seen in the proof of Theorem 4.10 , the case $w=2$ is problematic only in the case $d=2 w-1$, and the problem can easily be solved if we have another runner we can use. Hence the result of the theorem is also true for $w=2$ if we only suppose $2 w \leq d$. 
Furthermore, the case $w=2$ (which is fairly small) can in fact be studied on its own, and one can show that, for any $d>0$, the unipotent $d$-blocks of $G$ of weight 2 satisfy the Nakayama Conjecture (provided $F \geq 2$ ).

4. Since the $d$-weight of a unipotent $d$-block of $G$ is at most $n / d$, we see that, if $n \leq d(d+1) / 2$, then all the unipotent $d$-blocks of $G$ satisfy the Nakayama Conjecture (provided $F \geq n / d$ ).

\subsection{And then?}

The proof of the Nakayama Conjecture in the general case seems to be hard. In $[8$, the authors prove that, if $B$ is an $\ell$-block of weight $w$ of some symmetric group, then there is a generalized perfect isometry between $B$ and the set of irreducible (complex) characters of the wreath product $\mathbf{Z}_{\ell} \succ S_{w}$. This means that the restricted scalar products of characters of $B$ across $\ell$-regular elements are, up to a sign, the same as the restricted scalar products of characters of $\mathbf{Z}_{\ell} \succ S_{w}$ across some (carefully chosen) set of elements (called regular elements).

It turns out that the computations are easier to carry out in the wreath product, where it can be shown that every character is directly linked across regular elements to the trivial character. Coming back to the symmetric group, one gets that any two characters in the combinatorial $\ell$-block are linked across $\ell$-regular elements. This proves that the combinatorial $\ell$-blocks and the $\ell$-blocks are the same, or, equivalently, that the $\ell$-blocks satisfy the Nakayama Conjecture.

Now, we might try and do something similar in the case of $G L(V)$. This would involve finding the relevant wreath product, and the set of conjugacy classes we want to distinguish in it. Given a combinatorial unipotent $d$-block of weight $w$, a natural candidate for the wreath product is $G L(d, q) 2 S_{w}$. However, even in easy cases (like $d=1$ ), this doesn't seem to contain enough information (in particular, it is hard to have powers of $q$ appearing).

So far, we can only conjecture that, for any $d>0$, the unipotent $d$-blocks of $G$ satisfy an analogue of the Nakayama Conjecture.

\section{Second Main Theorem}

One of the very striking properties of ordinary blocks of finite groups is Brauer's Second Main Theorem (cf e.g. [10). In 8, the authors give an analogue of this in the context of generalized blocks. Depending on the generalized sections we define, the blocks we obtain may satisfy this analogue or not.

\subsection{Domination, Second Main Theorem Property}

For all the definitions and results in this section, we refer to 8 . We start by defining blocks for the centralizers of $d$-elements. Recall that the $d$-blocks of $G$ are defined by orthogonality across the set $\mathcal{Y}_{d}(1)$ of $d$-regular elements. As a consequence, they separate $\mathcal{Y}_{d}(1)$ from its complement (cf $[8$, Corollary 1.2). For $x \in \mathcal{X}_{d}$, we define the $d$-blocks of $C_{G}(x)$ to be the smallest (non-empty) subsets of $\operatorname{Irr}\left(C_{G}(x)\right)$ such that irreducible characters in distinct subsets are orthogonal across $x \mathcal{Y}_{d}(x)$. We can equally define them to be non-empty subsets of $\operatorname{Irr}\left(C_{G}(x)\right)$ which are minimal subject to separating $\mathcal{Y}_{d}(x)$ from its complement 
in $C_{G}(x)$ (or, equivalently, to separating $x \mathcal{Y}_{d}(x)$ from its complement (since $x$ is central in $\left.\left.C_{G}(x)\right)\right)$.

We now turn to the notion of domination. Suppose $\chi \in \operatorname{Irr}(G)$ and $\beta$ is a union of $d$-blocks of $C_{G}(x)$ for some $x \in \mathcal{X}_{d}$. We define a generalized character $\chi^{(\beta)}$ of $C_{G}(x)$ via

$$
\chi^{(\beta)}=\sum_{\mu \in \beta}<\operatorname{Res}_{C_{G}(x)}^{G}(\chi), \mu>\mu .
$$

Definition 5.1. Let $x \in \mathcal{X}_{d}$ and $b$ be a d-block of $C_{G}(x)$. We say that a d-block $B$ of $G$ dominates $b$ if there exist $\chi \in B$ and $y \in \mathcal{Y}_{d}(x)$ such that $\chi^{(b)}(x y) \neq 0$.

We see that, for $x \in \mathcal{X}_{d}$, if $\chi \in B$ for some $d$-block $B$ of $G$, then, for each $y \in \mathcal{Y}_{d}(x)$, we have $\chi(x y)=\sum_{b} \chi^{(b)}(x y)$, where $b$ runs through the set of $d$-blocks of $C_{G}(x)$ dominated by $B$.

Note that, for $x \in \mathcal{X}_{d}$, each $d$-block of $C_{G}(x)$ is dominated by at least one $d$-block of $G$.

Definition 5.2. We say that the d-blocks of $G$ satisfy the Second Main Theorem Property if, for each $x \in \mathcal{X}_{d}$ and each $d$-block $b$ of $C_{G}(x), b$ is dominated by a unique d-block of $G$.

Note that, if, instead of $\mathcal{X}_{d}$, we take the set of $r$-elements of $G$ ( $r$ a prime), and, instead of $\mathcal{Y}_{d}(x)$, we take the set of $r$-regular elements of $C_{G}(x)$, then we obtain the $r$-blocks of $G$, and they do satisfy the Second Main Theorem Property.

Using the fact that, for $x \in \mathcal{X}_{d}$, irreducible characters of $C_{G}(x)$ in distinct $d$-blocks are orthogonal across $x \mathcal{Y}_{d}(x)$, one proves easily the following:

Proposition 5.3. ([8], Corollary 2.2) The d-blocks of $G$ satisfy the Second Main Theorem Property if and only if, for each d-block $B$ of $G$, there is, for each $x \in \mathcal{X}_{d}, a$ (possibly empty) union $\beta(x, B)$ of d-blocks of $C_{G}(x)$ such that, for each irreducible character $\chi \in B$ and each character $\mu \in \beta(x, B)$, we may find a complex number $c_{\chi, \mu}$ such that, for each $y \in \mathcal{Y}_{d}(x)$, we have

$$
\chi(x y)=\sum_{\mu \in \beta(x, B)} c_{\chi, \mu} \mu(x y),
$$

and, furthermore, $\beta(x, B)$ and $\beta\left(x, B^{\prime}\right)$ are disjoint whenever $B$ and $B^{\prime}$ are distinct d-blocks of $G$.

The following theorem enlighten the link between the Second Main Theorem Property and Brauer's Second Main Theorem:

Theorem 5.4. ([8], Corollary 2.3) Suppose that the d-blocks of $G$ satisfy the Second Main Theorem property. Then:

(i) Irreducible characters of $G$ which are in distinct d-blocks are orthogonal across each $\mathcal{Y}_{d}$-section of $G$.

(ii) If $x \in \mathcal{X}_{d}$ and $\sum_{\chi \in \operatorname{Irr}(G)} a_{\chi} \chi$ is a class function which vanishes identically on the $\mathcal{Y}_{d}$-section of $x$ in $G$, then, for each $d$-block $B$ of $G, \sum_{\chi \in B} a_{\chi} \chi$ also vanishes identically on the $\mathcal{Y}_{d}$-section of $x$ in $G$.

(iii) d-blocks of $G$ separate $\mathcal{Y}_{d}$-sections of $G$. 


\subsection{Second Main Theorem Property for combinatorial unipo- tent $d$-blocks}

We first study the $d$-blocks of the centralizers of the $d$-elements. If $x \in \mathcal{X}_{d}$ has $d$-type $\mathbf{k m}$, then, writing $l=n-\mathbf{k m} d$, we have $C_{G}(x)=H_{0} \times H_{1}$ where $H_{1} \cong$ $G L(l, q)$ and $H_{0} \leq G_{0} \cong G L(\mathbf{k m} d, q)$. Then $\operatorname{Irr}\left(C_{G}(x)\right)=\operatorname{Irr}\left(H_{0}\right) \otimes \operatorname{Irr}\left(H_{1}\right)$. Note that, as we noted before, we may consider $x$ as an element of $H_{0}$, and then $x \mathcal{Y}_{d}(x)=\left\{(x, y) \in H_{0} \times H_{1}, y \in \mathcal{Y}_{d}^{l}(1)\right\}$.

Take $\chi_{0}, \psi_{0} \in \operatorname{Irr}\left(H_{0}\right)$ and $\chi_{1}, \psi_{1} \in \operatorname{Irr}\left(H_{1}\right)$. We have

$$
\begin{aligned}
<\chi_{0} \otimes \chi_{1}, \psi_{0} \otimes \psi_{1}>_{x} \mathcal{Y}_{d}(x) & =\frac{1}{\left|C_{G}(x)\right|} \sum_{y \in \mathcal{Y}_{d}(x)}\left(\chi_{0} \otimes \chi_{1}\right)(x y) \overline{\left(\psi_{0} \otimes \psi_{1}\right)(x y)} \\
& =\frac{1}{\left|C_{G}(x)\right|} \sum_{y \in \mathcal{Y}_{d}^{l}(1)} \chi_{0}(x) \chi_{1}(y) \overline{\psi_{0}(x) \psi_{1}(y)} \\
& =\frac{\chi_{0}(x) \overline{\psi_{0}(x)}}{\left|C_{G}(x)\right|} \sum_{y \in \mathcal{Y}_{d}^{l}(1)} \chi_{1}(y) \overline{\psi_{1}(y)} \\
& =\frac{\chi_{0}(x) \overline{\psi_{0}(x)}}{\left|H_{0}\right|}<\chi_{1}, \psi_{1}>_{\mathcal{Y}_{d}^{l}(1)} .
\end{aligned}
$$

Since $x$ is central in $H_{0}$, we have $\chi_{0}(x) \overline{\psi_{0}(x)} \neq 0$, and we see that $\chi_{0} \otimes \chi_{1}$ and $\psi_{0} \otimes \psi_{1}$ are directly $x \mathcal{Y}_{d}(x)$-linked if and only if $\chi_{1}$ and $\psi_{1}$ are directly $\mathcal{Y}_{d}^{l}(1)$ linked. Extending by transitivity, we obtain that the $d$-blocks of $C_{G}(x)$ are the $\operatorname{Irr}\left(H_{0}\right) \otimes b_{i}$ 's, where $b_{i}$ runs through the set of $\mathcal{Y}_{d}^{l}(1)$-blocks (i.e. $d$-blocks) of $H_{1} \cong G L(l, q)$.

In analogy with this, we define the unipotent $d$-blocks and combinatorial unipotent $d$-blocks of $C_{G}(x)$ to be the $\operatorname{Irr}\left(H_{0}\right) \otimes b_{i}$ 's, where $b_{i}$ runs through the sets of unipotent $d$-blocks and combinatorial unipotent $d$-blocks of $H_{1} \cong G L(l, q)$ respectively.

We can now prove that the combinatorial unipotent $d$-blocks of $G$ satisfy the Second Main Theorem Property.

Take any $x \in \mathcal{X}_{d}$, and write $C_{G}(x)=H_{0} \times H_{1}$ as above. For any combinatorial unipotent $d$-block $B$ of $G$, labeled by the $d$-core $\gamma$, we set $\beta(x, B)=$ $\operatorname{Irr}\left(H_{0}\right) \otimes b$, where $b$ is the combinatorial unipotent $d$-block of $H_{1}$ labeled by $\gamma$. For any $\chi_{\mu} \in B$ and $\psi_{0} \otimes \psi_{\lambda} \in \operatorname{Irr}\left(H_{0}\right) \otimes b$, we set

$$
c_{\chi_{\mu}, \psi_{0} \otimes \psi_{\lambda}}=\left\{\begin{array}{ll}
\alpha_{\mu \lambda}^{x} & \text { if } \psi_{0}=1_{H_{0}} \\
0 & \text { otherwise }
\end{array},\right.
$$

where the $\alpha_{\mu \lambda}^{x}$ 's are the MN-coefficients, obtained from the Murnaghan-Nakayama rule for unipotent characters.

Then the definition of the $\alpha_{\mu \lambda}^{x}$ 's shows that, for each $x \in \mathcal{X}_{d}$, the $\beta(x, B)$ 's and $c_{\chi_{\mu}, \psi_{0} \otimes \psi_{\lambda}}$ 's satisfy the hypotheses of Proposition 5.3. Indeed, for each combinatorial unipotent $d$-block $B$ of $G$, for each $\chi_{\mu} \in B$ and for each $y \in \mathcal{Y}_{d}(x)$, we have

$$
\chi_{\mu}(x y)=\sum_{\psi_{0} \otimes \psi_{\lambda} \in \beta(x, B)} c_{\chi_{\mu}, \psi_{0} \otimes \psi_{\lambda}}\left(\psi_{0} \otimes \psi_{\lambda}\right)(x y),
$$

and, furthermore, $\beta(x, B)$ and $\beta\left(x, B^{\prime}\right)$ are disjoint whenever $B$ and $B^{\prime}$ are distinct combinatorial unipotent $d$-blocks of $G$. This implies that, for each 
$x \in \mathcal{X}_{d}$, each combinatorial unipotent $d$-block of $C_{G}(x)$ is dominated by a unique combinatorial unipotent $d$-block of $G$. This proves the following:

Theorem 5.5. For any positive integers $n$ and $d$, the combinatorial unipotent $d$-blocks of $G$ satisfy the Second Main Theorem Property. In particular, if $d=1$, then the unipotent d-blocks of $G$ satisfy the Second Main Theorem Property, and, for any $d>0$, the unipotent $d$-blocks of $G$ of weight 0,1 , 2, and at most $(d+1) / 2$ satisfy the Second Main Theorem Property.

Remark: It is easy to see that one can change the definition of $d$-regular element to "an element whose minimal polynomial has no irreducible factor of degree $d$ (except maybe $X-1$ )" (instead of "degree divisible by $d$ ") without affecting any of the results we have proved, apart from Theorem 4.5 (Nakayama Conjecture in the case $d=1$ ).

\section{Acknowledgements:}

I wish to thank deeply Meinolf Geck, Radha Kessar, Attila Maróti and Geoffrey R. Robinson for the many discussions we had about this work. I also wish to thank Paul Fong and Bhama Srinivasan for their kindness and support. Finally, I thank Olivier Mathieu for his help with the proof of Lemma 4.9.

\section{References}

[1] D. Alvis, The duality operation in the character ring of a finite Chevalley group, Bull. Amer. Math. Soc. (N.S.) 1(6), 907-911 (1979).

[2] C. W. Curtis, Reduction theorems for characters of finite groups of Lie type, J. Math. Soc. Japan 27(4), 666-688 (1975).

[3] F. Digne, J. Michel, Foncteurs de Lusztig et caractères des groupes linéaires et unitaires sur un corps fini, J. Algebra 107, 217-255 (1987).

[4] H. Farahat, On the representations of the symmetric group, Proc. London Math. Soc. 4, 303-316 (1954).

[5] P. Fong, B. Srinivasan, The blocks of finite general linear and unitary groups, Invent. Math. 69, 109-153 (1982).

[6] J. A. Green, The characters of the finite general linear groups, Trans. Amer. Math. Soc. 80, 402-447 (1955).

[7] G. James, A. Kerber, The representation theory of the symmetric groups, Encyclopedia of Mathematics and its Application 16, Addison-Wesley (1981).

[8] B. Külshammer, J. B. Olsson, G. R. Robinson, Generalized blocks for symmetric groups, Invent. Math. 151(3), 513-552 (2003).

[9] A. Maróti, On generalized blocks for alternating groups, J. Algebra 297, 400-408 (2006).

[10] G. Navarro, Characters and blocks of finite groups, Lond. Math. Soc. Lecture Note Series 250, Cambridge University Press (1998). 